\title{
Mouvements de résonance à deux dimensions dans une enceinte sous l'action d'ondes incidentes
}

\author{
Two dimensional resonant motion \\ in basins subjected to incident waves
}

\author{
par F. BIESEL ET B. LE MÉHAUTÉ
}

INGÉNELURS A LA SOGREAH

\begin{abstract}
L'amplitude des seiches dans les darses, sontmises à l'agitation du large et dans lesquelles les mouvements sont pratiquement bi-dimensionnels, dépend des formes d'entrée par lesquelles agit l'excitation.

Les différents types de darses peuvent donc être classés suivant le type « d'obstacles 》 caractérisant ces formes d'entrée. Cet article fait suite $\grave{a}$ « l'étude théorique de la houle sur certains obstacles 》dont les résultats sont directement utilisés.

Les essais vérifient les théories de la résonance basées sur ces principes (darses \& sousouvertes », darses en série) et suppléent aux cas ne pouvant être analysés (darses « sur-oubertes »). L'agitation dans les darses limitées par un ouvrage perméable est anussi étudiée (et vérifiée expérimentalement) por la théorie des obstacles résistants. Ceci conduit à définir la «pseudo-résonance ».

L'influence des frottements est examinée expérimentalement et permet de tirer des conclusions pratiques pour la technique du modèle réduit de seiches portuaires.

Ces études se terminent sur une synthèse des lois de résonance des mouvements plans par l'introduction de la notion de « degré d'ouverture ». Les lois émises s'appliquent également $\dot{a}$ tous les types d'ondes de gravité (marées, seiches et houles) quelle que soit leur période.
\end{abstract}

In basins subjected to "agitation" from the open sea, and in which motion is to all intents and purposes two dimensional, the amplitude of seiches is dependent upon the types of entries through which disfurbance is transmitted.

We may therefore classify various types of basins according to the type of "obstacle" that may be taken to represent the basin entry. This article is a continuation of "The Theoretical Study of the Reflection of Waves from different Types of Obstacles", and the results of this study are used directly.

Experiments bear out resonance theories based on these principles (restricted opening basins, basins in series) and fill in the gaps in cases that cannot be analysed (expanded opening basins).

Agitation in basins bounded by permeable structures is also investigated (and checked experimentally) making use of the theory of the resistance of obstacles.

The part played by friction is studied experimentälly and leads to results useful in scale model techniques applied to harbour seiches. To conclude these investigations, laws of resonance for two dimensional motions are synthesized by introducing the idea of "degree of opening". The resulting laws are equally applicable to all kinds of gravity waves (tides, seiches and swell), whatever their period.

\section{A. - GÉNÉRALITÉS}

\section{I. - Avant-propos}

Les mouvements de seiches dans les lacs ont été depuis longtemps soumis aux calculs des théoriciens et expérimentateurs les plus compétents.

Les études des seiches dans les bassins ouverts soumis à l'action d'ondes venant du large sont moins nombreuses et surtout moins rigoureuses. Cependant on peut citer celles de lord Rayleigh, celles de Honda, Terada, Yoshida, Isitani [6], celles de Hansen, les travaux importants de Proudman [11] sur les marées qui présentent souvent des caractères communs avec les seiches. 
ceux de NEUMANN [10] introduisant la notion d'impédance en hydraulique, de LAMOËN $\mid 7\rceil$ sur la théorie des estuaires, etc... Plus récemment, Mc Nown a étudié théoriquement et expérimentalement au Laboratoire Dauphinois d'Hydraulique les cas des ports circulaires et carrés [9], étude complétée par MM. KRavtrchenko et Ápté [15] [16].

Sur le plan purement théorique, on connaît depuis longtemps des formules donnant la période de résonance de bassins ouverts ou fermés, de forme suffisamment allongée pour que l'on puisse y considérer que les mouvements d'oscillation se font uniquement dans le sens de leur longueur. Par analogie avec la théorie classique des tuyaux sonores, on n'a considéré en général que deux cas: celui des bassins complètement fermés et celui des bassins complètement ouverts. Les périodes de résonance sont alors données en principe par :

$$
\mathrm{T}=\frac{2}{k+1} \int_{0}^{d} \frac{d x}{\sqrt{g h(x)}}
$$

pour les bassins fermés ou ouverts aux deux extrémités (fig. $1 a$ et $1 b$ );

$$
\mathrm{T}=\frac{4}{2 k+1} \int_{0}^{d} \frac{d x}{\sqrt{g h(x)}}
$$

pour les bassins ouverts à une extrémịté seulement (fig. $1 \mathrm{c}$ );

$k$ étant un nombre entier caractérisant l'harmonique,

$d$ étant la longueur du bassin prise dans le sens du mouvement sur l'axe médian de la surface libre;

$h(x)$ étant la profondeur moyenne dans un plan perpendiculaire au mouvement et d'abscisse $x$.

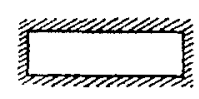

a)

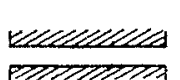

b)

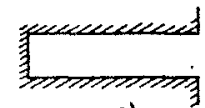

c)

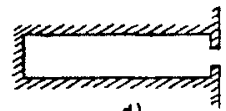

d)
FIG. 1. - Différents types de darses.

Ces formules supposent qu'il ne peut y avoir de réflexion d'ondes qu'aux extrémités du bassin (avec ou sans changement de signe suivant que la réflexion se fait sur une paroi ou sur un élargissement ou approfondissement brusque). Les sections transversales ne doivent donc varier que graduellement à l'intérieur du' bassin.

De ces formules, on déduit qu'un bassin fermé contient $(k+1)$ fois la demi-longueur d'onde, alors qu'un bassin ouvert contient $[(2 k+1) / 2]$ fois cette même quantité. On dit, par analogie acoustique, qu'un bassin fermé résonne en $1 / 2$ onde et un bassin ouvert en $1 / 4$ d'onde.

La présente étude s'efforce de préciser ces notions et d'établir les lois de résonance en fonc- tion du type et de la largeur de la passe d'entrée (fig. $1 d$ ). Elle se limite aux mouvements à deux dimensions dans le bassin étudié. Les courants au voisinage de la passe d'entré, bien que localement à trois dimensions, se transforment en effet très rapidement en un mouvement plan, dès que l'on s'éloigne de cette passe. La théorie a été établie et les essais ont été effectués pour une darse rectangulaire de piofondeur constante. Les résultats trouvés et les lois émises présentent toutefois un caractère de plus grande généralité; à l'aide du calcul numérique et de formules analogues'à celles cilées cidessus, ils peuvent s'appliquer à tout mouvement se faisant, dans un port allongé de sections graduellement variées, suivant l'axe de la passe d'entrée.

Nous donnons tout d'abord quelques définitions sur les différents types de darses pouvant se présenter, définitions que nous utiliserons dans cette étude.

\section{II. - Différents types de darses}

(Figure 2)

Les définitions proposées ci-dessous peuvent sembler arbitraires. En fait, elles nous ont été dictées par l'étude des obstacles soumis à la

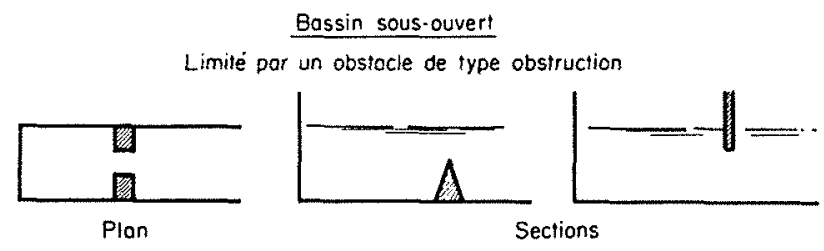

Bossin sur-ouvert

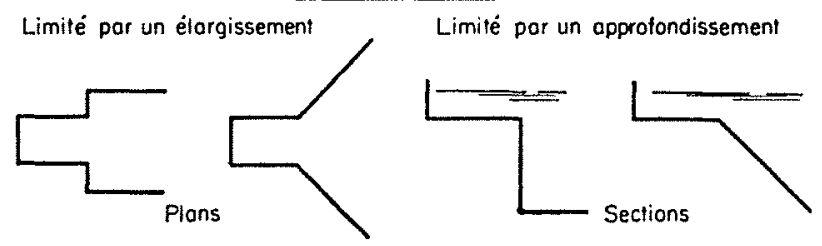

Bossin limite por un obstacle complexe

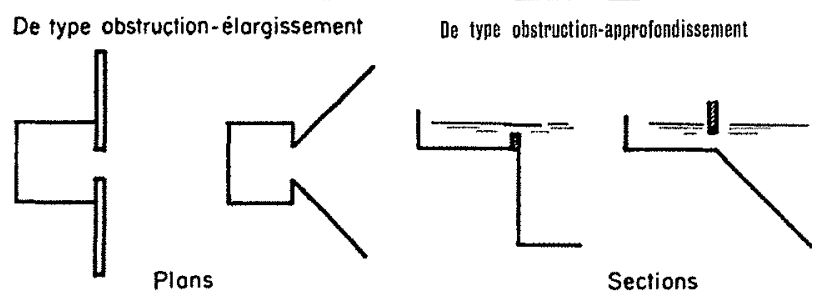

Fig. 2. - Différents types de darses classés suivant leurs conditions de résonance. 
houle, étude dont les résultats théoriques s'appliquent directement aux seiches [1 et 3].

1. Une darse est dite « sous-ouverte» lorsqu'elle est limitée par un obstacle du type «obstruction » et qu'elle débouche sur un canal de même largeur et de même profondeur. L'obstacle du type « obstruction » réalisera une restriction du passage offert aux houles. Ce sera essentiellement un objet disposé sur le passage des ondes.

2. Une darse est dite « sur-ouverte » lorsqu'elle est limitée :

a) par un obstacle du type « élargissement » caractérisé par une profondeur constante et une augmentation brusque de la largeur vers le large;

b) par un obstacle du type « approfondissement » caractérisé par une largeur constante et une augmentation brusque de la profondeur vers le large.

L'obstacle «simple » ne répond qu'à une des caractéristiques précitées. L'obstacle « complexe » est composé de plusieurs obstacles simples, superposés sur une longueur très faible (en général de l'ordre de grandeur de la profondeur dans la darse).

On peut, par suite, classer les obstacles complexes en trois catégories : :

- obstacles du type élargissement et approfondissement,
- obstacles du type obstruction el approfondissement,

- obstacles du type approfondissement, élargissement et obstruction.

La « courbe d'agitation» donne la valeur de l'agitation rapportée à l'amplitude de l'onde incidente en fonction de la longueur de la darse exprimée en longueur d'onde.

Nous dirons qu'il y a résonance lorsque le rapport de l'agitation dans la darse à l'amplitude incidente passe par un maximum lorsque varie la fréquence. Remarquons que cette définition diffère de celle donnée par le professeur MacNown qui appelait périodes de résonance celles suivant lesquelles se faisaient les mouvements propres des plans d'eau limités à l'entrée par une condition géométrique simple. Pour étudier l'influence de la passe d'entrée sur les conditions de résonance et sur la «correction d'ouverture », il est préférable d'adopter une définition plus générale, telle que celle énoncée cidessus.

\section{III. - Appareillage expérimental}

Les essais ont été effectués aternativement tans deux canaux à houles, suivant les possibilités pratiques qu'ils ofrraient aux différentes mesures.
Fig. 3.

Installations d'essais :

1. Ouvrages absorbants,

2. Rail de mesure de l'enregistreur graphique de houle,

3. Limite du canal,

4. Fond de la darse,

5. Darse,

6. Fntrée de la darse,

7. Blocs parallélépipédiques $(40 \times 28 \times 8)$

8. Ecrou de fixation du rail,

9. Canal extérieur.

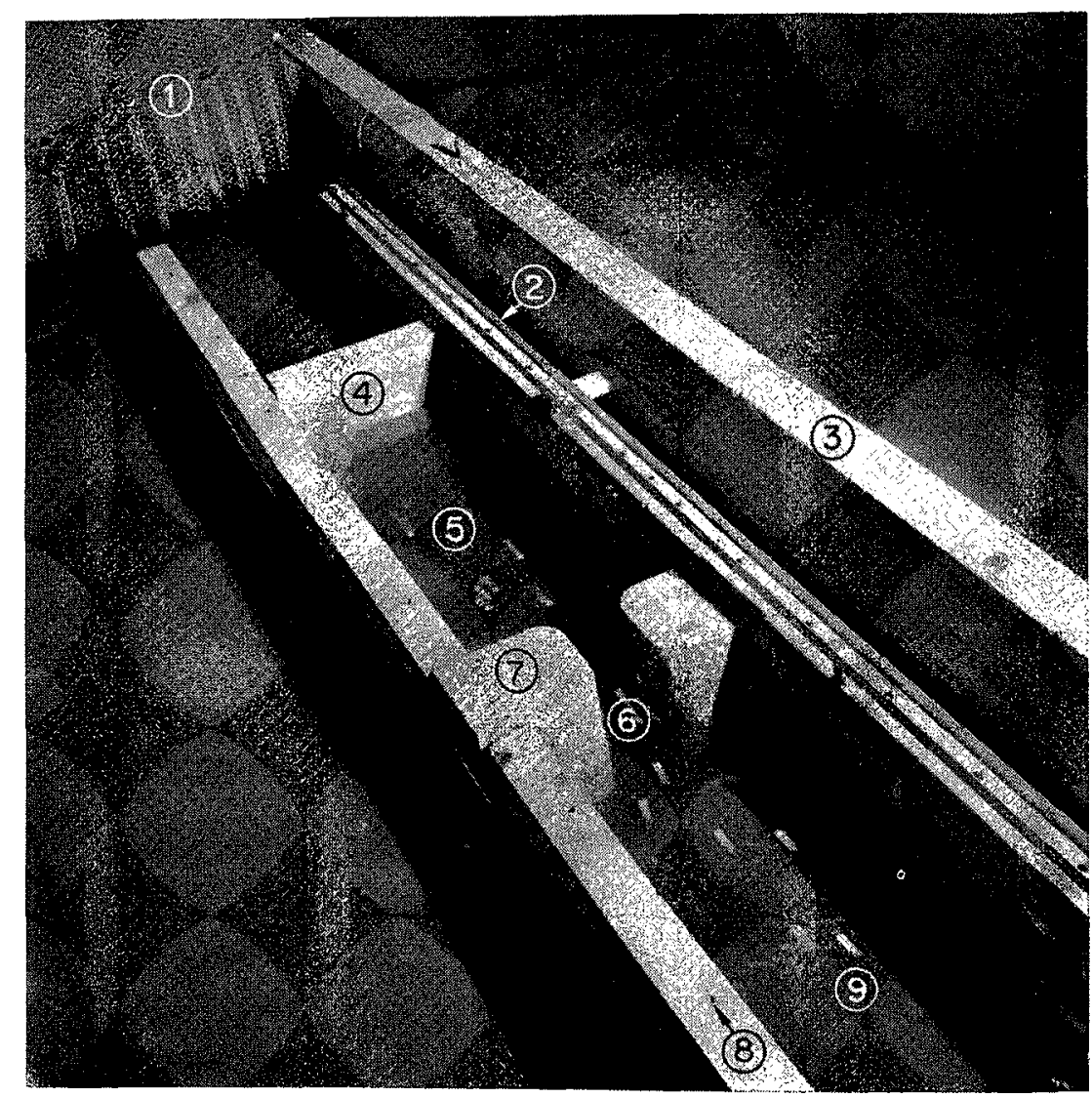


Le premier canal ulilisé est une dalle de $24 \mathrm{~m}$ sur $4 \mathrm{~m}$, nivelée au $\mathrm{mm}$ près. Des blocs parallélépipédiques préfabriqués $(40 \times 28 \times 8)$ permettent de construire très rapidement les darses nécessaires. Le générateur de houle du type «balancoire» [12] permet de réaliser des périodes de 0,5 à 3 secondes. Une grande épaisseur $(3 \mathrm{~m})$ de filtre à houle en grillage permet d'épurer la houle et de limiter les effets de résonance propres à l'ensemble du bassin.

ree second canal utilisé, d'une largeur de $30 \mathrm{~cm}$, n'a permis d'effectuer que des essais à deux dimensions. Par contre, ses parois étant vitrées, il permet de mieux se rendre compte des phénomènes. Le généraleur de houle du canal, egalement du type « balançoire », permet de faire varier les périodes de 0,4 à 2,8 secondes.

Les mesures d'amplitude ont été effectuées par observations directes sur la vitre à la pointe de mesure à wil cathodicque, à l'enregistreur d'onde à capacité, et à l'enregistreur graphique de houle (E.G.H.). Ces apparcils ont été décrits dans la Houille Blanche [5]. Le rail nécessaire au déplacement de la sonde à capacité de l'E.G.H. est fixé sur les blocs de béton limitant la darse ou le canal d'essais (fig. 3). Les périodes sont déterminées au chronomètre.

\section{B. - DARSE SOUS OUVERTE}

\section{I. - Théorie}

La théorie établissant la valeur de l'agitation entre deux obstacles identiques est particulièrement intéressante pour l'étude des seiches [2] [8], car elle reste valable pour une darse telle que celle de la figure 4 qui peut être considérée comme étant limitée par un obstacle parfaitement réfléchissant (le mur de fond) et un obstacle quelconque (l'entrée).

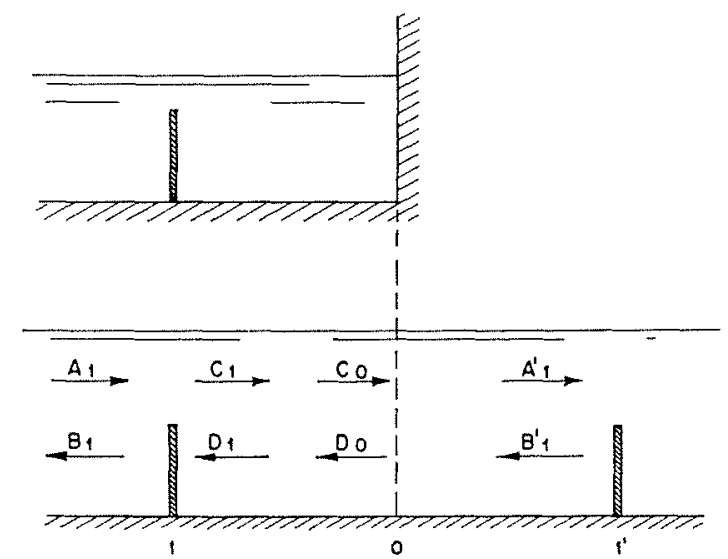

Frti. 4. - L'application de la méthode des images permel de ramener l'étude du mouvement dans une darse à celle du mouvement entre deux obstacles identiques.

Nous avons vu en effet [2] que tout obstacle, quelle que soit sa forme géométrique, ne présentait aucune dissymctrie à la houle. I'obstacle se trouvant à l'entrée de la darse a donc les mëmes caractéristiques $\alpha$ et $\beta$, pour la houle incidente, et la houle réfléchie en 0 sur le fond de la darse
( $\alpha$ et $\beta$ sont respectivement les coefficients de transmission et de réflexion de l'obstacle).

L'application de la méthode des images permet donc de ramener l'étude du mouvement dans une telle darse à celle du mouvement entre deux obstacles identiques, dont l'un serait l'entrée de la darse et l'autre l'image de ce dernier par rapport au mur du fond. Les ćquations établies dans la théorie des obstacles soumis à la houle restent donc valables, à condition de diviser les distances entre les obstacles par deux et de multiplier les amplitudes maxima par deux.

Dans le cas simple d'une darse sous-ouverte donnant sur un canal de même largeur et de même profondeur, le mouvement à trois dimcnsions au voisinage de l'entrée se traduit en fait par un mouvement à deux dimensions dans la darse. Il est possible d'obtenir directement l'expression de l'agitation sans faire appel aux calculs précédemment cités. On peut écrire tout d'abord :

$$
\begin{aligned}
& \mathrm{C}_{1}=\ddot{\alpha} \mathrm{A}_{1}+\bar{\beta} \mathrm{D}_{1} \\
& \mathrm{~B}_{1}=\bar{\alpha} \mathrm{D}_{1}+\overline{\bar{\beta}} \mathrm{A}_{1} \\
& \mathrm{C}_{1}=\bar{r}^{1 / 2} \mathrm{C}_{0} \\
& \mathrm{D}_{0}=\ddot{r}^{1 / 2} \mathrm{D}_{1} \\
& \mathrm{C}_{0}=\mathrm{D}_{0}
\end{aligned}
$$

avec :

$$
\bar{r}=e^{i \theta}
$$

et :

$$
\overline{0}=\frac{2 \pi d}{\mathrm{~L} / 2}
$$

$d$ étant la longueur de la darse et $\mathrm{L}$ la longueur d'onde localc. 
De ces équations, on tire :

$$
\begin{aligned}
& \mathrm{B}_{1}=\left[\frac{\bar{\alpha}^{2}}{\bar{r}-\bar{\beta}}+\bar{\beta}\right] \mathrm{A}_{1} \\
& \mathrm{C}_{1}=\frac{\bar{r} \bar{\alpha}}{\bar{r}-\dot{\beta}} \mathrm{A}_{1} \\
& \mathrm{D}_{1}=\frac{\bar{\alpha}}{\bar{r}-\bar{\beta}} \mathrm{A}_{1}
\end{aligned}
$$

Si Ag désigne l'agitation au ventre du mouvement mesurée au droit de l'obstacle 0 , on a :

d'où :

$$
\mathrm{A} g=\mathrm{C}_{0}+\mathrm{D}_{0}
$$

$$
\mathrm{A} g=\frac{2 \bar{\alpha} \cdot \bar{r}^{1 / 2}}{\overline{\bar{r}}-\bar{\beta}} \mathrm{A}_{1}
$$

Les courbes de résonance en $\mathrm{A} g$ doivent donc être les mêmes que celles trouvées lors de la théorie sur les obstacles [3] à un facteur 2 près (fig. 5).

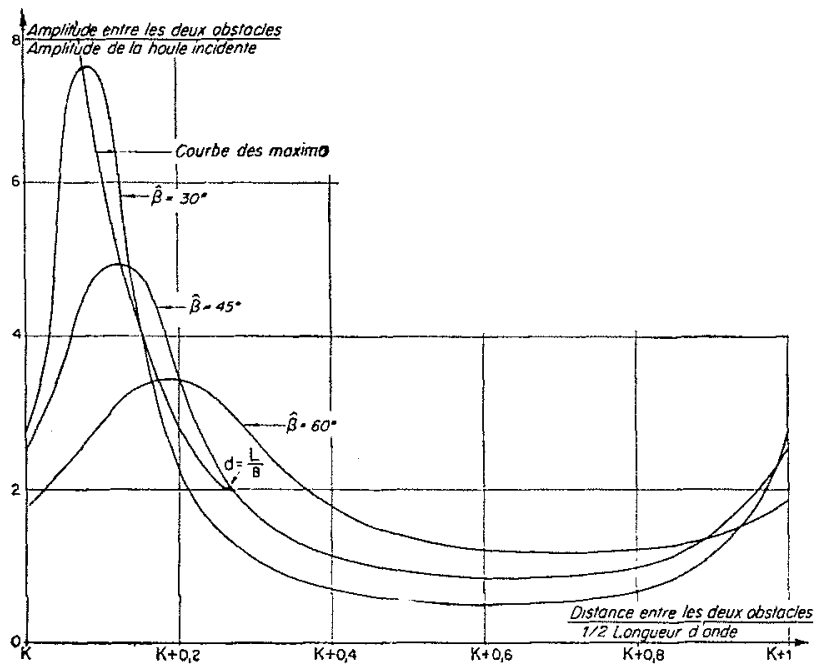

Fia. 5. - Agitation dans une darse sous-onverte. Courbes théoriçues calculées pour diverses valeurs du déphasage de l'onde réfléchie par rapport à l'onde incidente.

On a de même les égalités et relations suivantes, $A_{3}$ et $A_{m}$ représentant les agitations maximum et minimum en valeur absolue :

Agitations maxima : $A_{M}=\frac{2 \alpha A}{1-\beta}$

(condition de résonance : $\bar{\theta}=\hat{\beta}+k \pi$ )

Agitations minima : $A_{\mathrm{MI}}=\frac{2 \alpha A}{1+\beta}$

$$
\begin{gathered}
\frac{A_{M} X A_{m}}{A_{1}{ }^{2}}=\frac{4 \alpha^{2}}{1-\beta^{2}}=4 \\
\alpha=\frac{A_{M} \times A_{m}}{\left(A_{M}+A_{m}\right) A_{1}}
\end{gathered}
$$

$\bar{\beta}$ restant égal à :

$$
\bar{\beta}=\frac{A_{M L}-A_{m}}{A_{M}+A_{m}}
$$

Lorsque $\hat{\beta}$ varie de 0 à $\pi / 2$, la rèsonance a lieu quand la longueur de la darse $d_{\mathrm{x}}$ exprimée en longueur d'onde varie de :

$$
k(\mathrm{~L} / 2) \text { à } k(\mathrm{~L} / 2)+(\mathrm{L} / 8) \text {. }
$$

La résonance dans un port fermé est donc sensiblement du type $1 / 2$ onde, la longueur qu'il faut ajouter à $\mathrm{L} / 2$ étant une fonction croissante de $\hat{\beta}$, c'est-à-dire de l'ouverture. Lorsque le bassin tend à être entièrement ouvert sur un canal de même largeur, la longueur de résonance tend théoriquement vers :

$$
k \frac{\mathrm{L}}{2}+\frac{\mathrm{I}}{8}
$$

L’amplitude de résonance est alors égale à l'auplitude du clapotis.

Considérons maintenant la houle réfléchie par l'ensemble, au droit de l'entrée de la darse. Nous avons vu que la houle réfléchie $B_{1}$ au droit de l'obstacle est égale à :

$$
\mathrm{B}_{1}=\left[\bar{\beta}+\frac{\bar{\alpha}^{2}}{\bar{r}-\bar{\beta}}\right] \mathrm{A}_{1}
$$

On peut vérifier que :

et que :

$$
\left|B_{1}\right|=\left|A_{1}\right|
$$

$$
\widehat{B_{1}}-\widehat{A_{1}}==\hat{\beta}+2 \operatorname{Arctg}\left[\frac{\operatorname{tg}^{2}(\hat{\beta} / 2)}{\operatorname{tg}(\hat{\theta}-\hat{\beta} / 2)}\right]+2 k \pi
$$

(obstacles localisés sans perte d'énergie).

On en déduit que les ventres du clapotis extérieur sont à des distances :

$$
\left[k \pi+\frac{\hat{\beta}}{2}+\operatorname{Arctg}\left(\frac{\operatorname{tg}^{2}(\hat{\beta} / 2)}{\lg (0-\hat{\beta}) / 2}\right)\right] \frac{L}{2 \pi}
$$

de l'obstacle. Dans le cas de la résonance, $\overline{0}=\hat{\beta}$ à $k \pi$ près et $\widehat{B}_{1}-\widehat{A}_{1}=\hat{\beta}-\pi(+2 k \pi)$.

Les ventres du clapotis extérieur sont alors à des distances de l'obstacle égales à :

$$
\left(k \pi+\frac{\bar{\beta}}{2}+\frac{\pi}{2}\right) \frac{\mathrm{L}}{2 \pi}=(k \pi+\ddot{a}) \frac{\mathrm{L}}{2 \pi}
$$

En particuliex, quand l'ouverture tend vers zéro, $\widehat{\beta}$ tend vers zéro et $B_{1}$ est en opposition de phase avec $A_{1}$. Le clapotis extérieur tend done à avoir un noud au droit de l'entrée.

Il est intéressant de calculer la différence de phase (dans le temps) entre le clapotis extérieur 
$A_{1}+B_{1}$ et le clapotis intérieur $C_{1}+D_{1}$. Cette différence est donnée par l'expression :

$$
\operatorname{Arg} \frac{C_{1}+D_{1}}{A_{1}+B_{1}}
$$

$=\operatorname{Arg}\left[\frac{(1+\bar{r}) \bar{\alpha}}{\bar{r}-\bar{\beta}+\bar{r}-\bar{\beta}-\bar{\beta}^{2}+\bar{\alpha}^{2}}\right]$

$$
\begin{array}{r}
=\operatorname{Arg}[(1+\bar{r}) \vec{\alpha}]-\operatorname{Arg}[(\bar{r}-\bar{\beta}) \\
\left.\left.+\bar{r} \bar{\beta}-\bar{\beta}^{2}+\bar{\alpha}^{2}\right)\right]
\end{array}
$$

$=\frac{\bar{\theta}}{2}+\bar{\alpha}-\frac{\bar{\beta}}{2}+\hat{\beta}+\frac{\pi}{2}=k \pi$

Les clapotis intérieur et extérieur sont donc toujours en phase (l'opposition de phase et la coincidence de phase n'étant pas discernables si l'on ne spécifie pas les ventres considérés). Par exemple, lorsque l'ouverture tend vers zéro, la phase de l'agitation intérieure est : $A_{1}-\pi / 2$, tandis que la phase du clapotis extérieur est $A_{1}+\pi / 2$.

\section{II. - Vérification expérimentale}

Cette première série d'essais porte sur la recherche systématique de la valeur de l'agitation en fonction de la longueur de la darse et de son ouverture.

Afin de limiter les effets de frottement, l'am- plitude de l'onde incidente a été prise extrêmement faible. Car pour retrouver l'allure des phénomènes indiqués par la théorie précédente, il est essentiel de réaliser des conditions d'essais telles que les pertes d'énergie soient aussi faibles que possible. Cette condition a également conduit à faire la principale série d'essais avec une passe d'entrée profilée et d'une longueur non négligeable par rapport à la longueur d'onde, afin que le rapport des forces de frottement aux forces d'inertie soit aussi faible que possible.

Différentes sortes d'obstacles ont été successivement étudiées d'une façon systématique. Nous nous bornons à donner l’essentiel des résultats.

\section{a) Fermetune par musoirs profilés.}

Tout d'abord, nous avons modifié la largeur de la passe d'entrée à l'aide de deux musoirs en béton de $20 \mathrm{~cm}$ d'épaisseur, ofrant aux mouvements de l'eau des formes profilées (fig. 6). Les

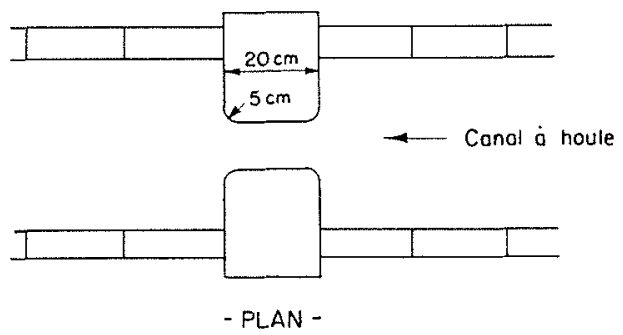

Fir. 6. - Type d'obstacle étudié.

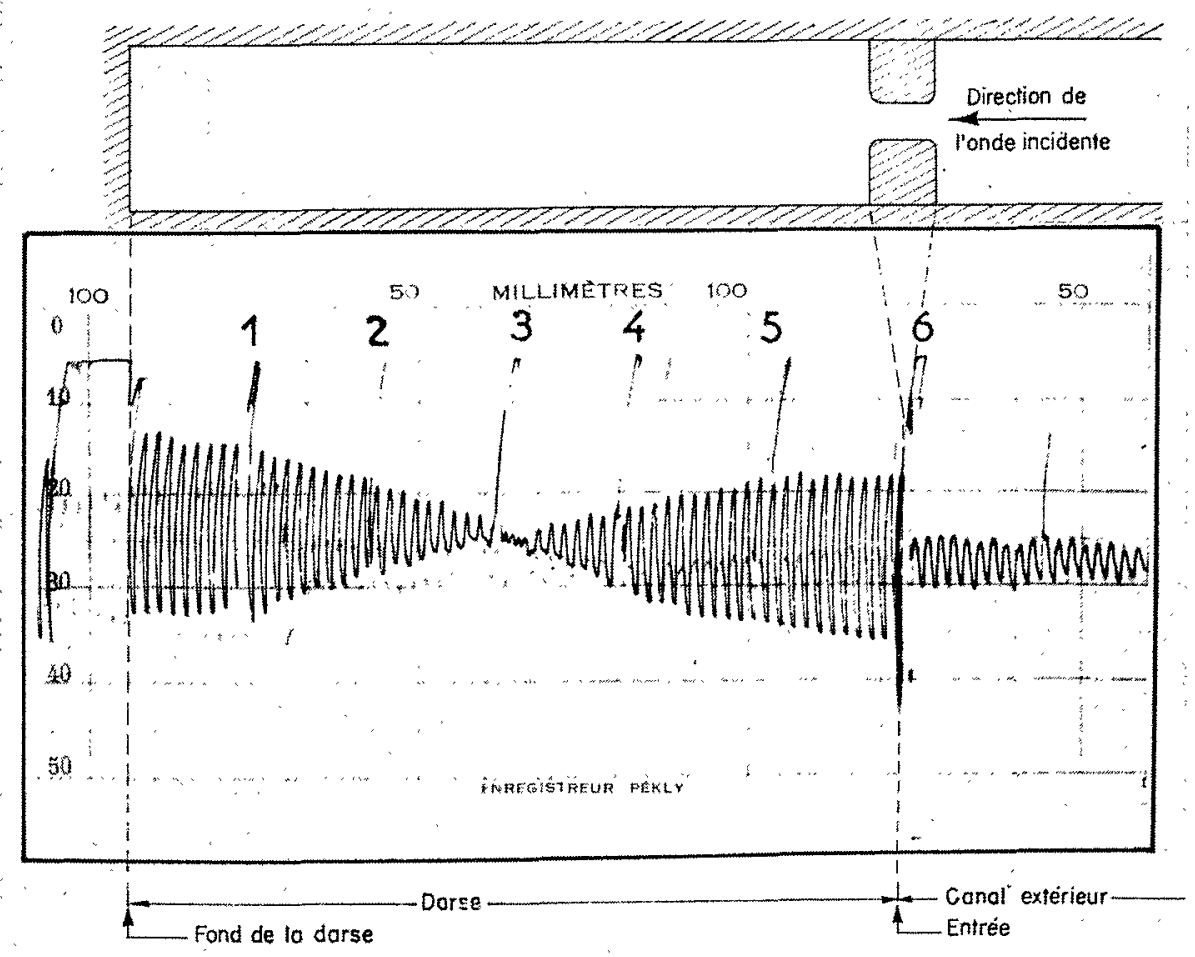

Fig. 7.

Agitalion dans une darse rectangulaire. Comparaison avee l'agitation au large. Distance entre tops: $20 \mathrm{~cm}$ modèle. 
mesures ont été faites à l'enregistreur graphique de houle le long de la darse et au large. Il nous a été ainsi possible de repérer les nœuds et les ventres du mouvement dans la darse et au large (fig. 7).

La figure 8 donne les résultats des essais d'agitation effectués dans les conditions suivantes:

- Période........ $\mathrm{T}=2 \mathrm{~s}$

- Amplitude. . . . . 2 $a=6,4 \mathrm{~mm}$

- Profondeur.....h $=140 \mathrm{~mm}$

Nous avons porté en abscisse la valeur du rapport de la longueur de la darse à la longueur d'onde dans la darse, et en ordonnée la valeur du rapport de l'agitation verticale maximum dans la darse à l'amplitude de l'onde incidente. La longueur $d$ de la darse est mesurée à partir de la face interne des blocs limitant la passe.

La concordance entre les résultats théoriques et expérimentaux est frappante. La courbe des maxima qui tend théoriquement vers l'infini pour une ouverture nulle, ne s'abaisse en effet qu'à partir d'une ouverture relative (rapport de l'ouvcrture $o$ à la largeur $l$ du canal), égale à 0,075 . De plus, la haute sélectivité des courbes d'agitation au voisinage de la résonance pour de faibles ouvertures permet de penser qu'il est encore possible d'accroître ce maximum, mais le réglage du modèle est alors extrêmement délicat,

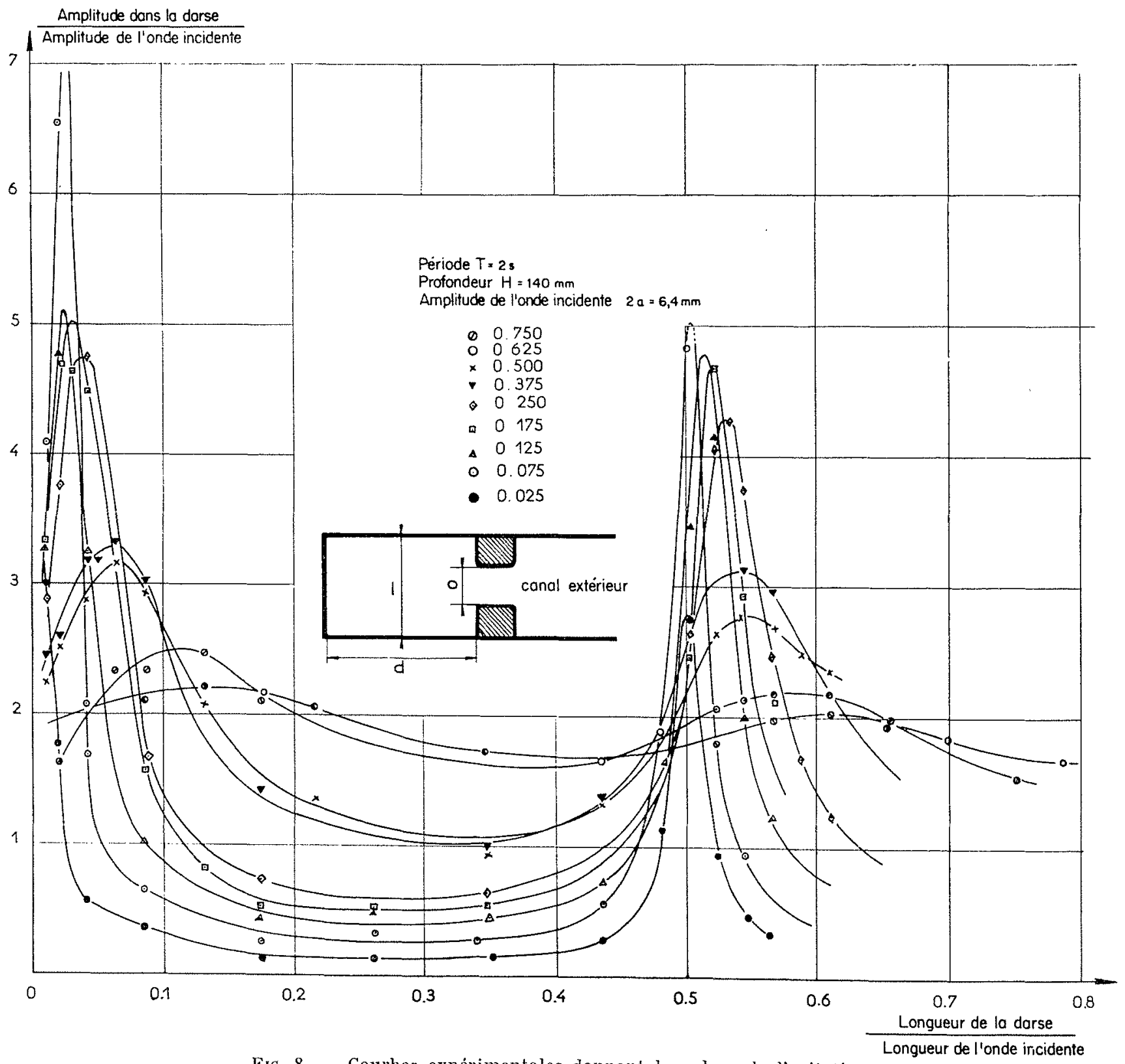

Frr. 8. - Courbes expérimentales donnant ha valeur de l'agitalion dons une darse sous-ouverte fermée par deux musoirs. 


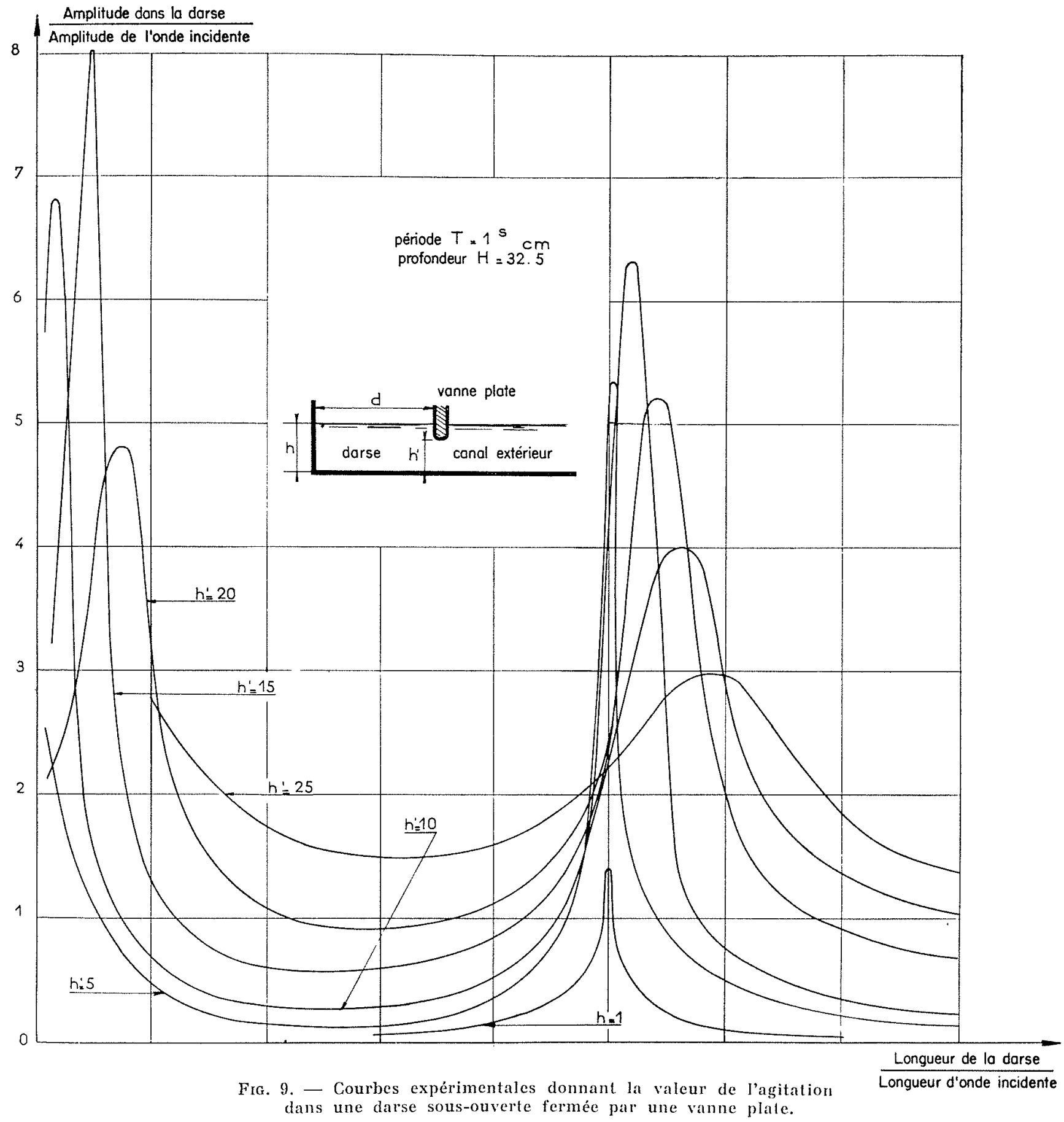

et nous n'arons pas pu dépasser dans ces conditions d'essai un maximum d'agitation égal à six fois l'amplitude de la houle extérieure.

Bien que l'ensemble des mesures n'ait porté que sur des longueurs de darse comprises entre 0 et 0,7 fois la longueur d'onde, nous avons vérifié sur quelques cas que, lorsqu'on augnente la longueur de la darse, on retrouve les conditions de résonance périodiquement, toutes les demi-longueurs d'onde. Cependant, les amplitudes atteintes tendent à décroître par suite de l'auginenta- tion des pertes d'énergie par frottements qui se produisent sur une longueur plus grande.

A l'opposé, nous avons vérifié, avec une certaine surprise, que l'allure périodique des courbes de résonance se conserve même jusqu'aux longueurs de darses extrêmement faibles [darses de longueur réduite à $(\widehat{\beta} / 2) \times(L / 2 \pi)]$. On aurait pu s'attendre, pour ce dernier cas, à ce que la théorie, basce sur les propriétés des ondes loin des ohstacles, devienne complètement fausse. Il est toutefois probahle que la longueur de lobs- 
tacle ait, dans ce cas, une certaine influence sur les conditions de résonance.

D'autres mesures ont été faites avec ces mêmes obstacles en faisant varier la période de 0,5 à 3 secondes. Les résultats, portés sur un système d'axes analogues, ne font que confirmer les conclusions déjà acquises en ce qui concerne l'allure générale des courbes d'agitation. Au cours de ces essais, il nous a été possible d'obtenir une amplitude d'agitation quatorze fois supérieure à l'amplitude de l'onde incidente dans les conditions suivantes :

- Période de la houle........ $1 \mathrm{~s}$

- Amplitude de la houle......2 $a=8 \mathrm{~mm}$

- Ouverture relative de la darse. $(o / l)=0,075$

\section{b) Fermeture par vanne plate :}

Des essais identiques ont été eflectués en obturant la darse par une vanne mince (fig. 9).

Les conditions d'essai sont alors les suivantes :

- Période de la houle.....T $=1 \mathrm{~s}$

- Profondeur du canal. . . .h $=32,5 \mathrm{~cm}$

On désigne par $h^{\prime}$ la distance entre le fond du canal et le niveau inférieur de la vanne. La vanne a une épaisseur de $2,5 \mathrm{~cm}$ et peut donc ètre considérée comme un obstacle localisé.

Pour des raisons de commodité dans les manipulations, les mesures ont été faites pour des longueurs de darse $d$ constantes, et des ouvertures $h^{\prime}$ variables. La valeur du rapport de l'amplitude de l'agitation $\mathrm{Ag}$ à l'amplitude de la houle (2a) a donc tout d'abord été exprimée en fonction de $h^{\prime}$ pour différentes valeurs de la longueur $d$. Un changement d'axes nous a permis d'exprimer $\mathrm{Ag} / 2 a$ en fonction de $d / \mathrm{L}$ pour différentes valeurs de $h^{\prime}$ (fig. 9). Les points expérimentaux, étant établis pour des valeurs $h^{\prime}$ quelconques, n'ont pas été portés sur les courbes.

Les résultats obtenus dans les conditions précitées répondent aux mêmes lois que ceux obtenus au paragraphe précédent et confirment donc la valeur de la théorie. Ià encore les conditions de résonance, lorsque la darse a pour longueur $(\hat{\beta} / 2) \times(\mathrm{L} / 2 \pi)$, sont très sensiblèment les mêmes que lorsque cette longueur est de $[k(\mathrm{~L} / 2)+(\bar{\beta} / 2) \times(\mathrm{L} / 2 \pi)]$.

L'importance des résonances obtenues pour des longueurs de darse inférieures à $L / 8$ (cette limite correspondant à la valeur maximum atteinte lorsque $\bar{\beta}=\pi / 2$ ), montre l'intérêt éventuel des résonateurs à $(\hat{\beta} / 2)(\mathrm{L} / 2 \pi)$ pour l'absorption de l'énergie d'ondes incidentes (M. Valembors appelle ce type : «résonateur en charge » [14]). Nous reviendrons prochainement sur ce cas dans une étude concernant les moyens de limiter les effets des seiches. Il est curieux de constater que le balancement binodal ou multinodal de la darse de longueur $(\hat{\beta} / 2) \times(\mathrm{L} / 2 \pi)$ fait place à un mouvement de l'eau en « piston » présentant une surface libre rigoureusement horizontale (fig. 10).

La mesure des déphasages entre les niveaux de l'eau de part et d'autre de la vanne est donnée par l'analyse d'un film de $16 \mathrm{~mm}-16$ images/seconde - pour des longueurs de darses inférieures à $\mathrm{L} / 8$. Lorsque la vanne est très peu enfoncée, l'amplitude dans la darse est la même que l'amplitude extérieure et les agitations sont évidemment en phase. Lorsque la vanne est très enfoncée ( $h$ ' tendant vers 0 ), l'amplitude est sensiblement la même que l'amplitude à l'extérieur, mais le déphasage est supérieur à $\pi / 2$. Le passage à $\pi / 2$ a lieu pour une ouverture d'autant plus faible que la longueur de la darse est faible. Il semble que ce passage corresponde à l'amplitude maximum dans la darse, c'est-à-dire à la résonance.

La discordance avec les résultats théoriques (déphasage 0 ou $\pi$ ) peut probablement s'expliquer par Jes pertes d'énergies à l'intérieur de la darse. Ces pertes sont relativement grandes en cas de résonance. La houle réfléchie est donc très affaiblie et la houle incidente prépondérante; or, pour les faibles ouvertures, elle tend théoriquement à être en quadrature avec le clapotis intérieur.

\section{c) Fermeturf par musoirs plans.}

Considérons maintenant des essais faits sur une darse fermée seulement par des musoirs à faces planes de $3 \mathrm{~cm}$ d'épaisseur.

Ces essais portent sur la recherche systématique des maxima d'agitation en fonction de la largeur o de l'ouverture de la darse, et pour une longueur de la darse voisine de la demi-longueur d'onde.

La houle incidente a pour caractéristique :

$$
\begin{aligned}
\text { - Période......... } & =2 \mathrm{~s} \\
\text { - Amplitude...... } & =7 \mathrm{~mm} \\
\text { - Profondeur...... } & =165 \mathrm{~mm}
\end{aligned}
$$

L'agitation maximum a lieu pour une ouverture relative $o / l$ de 0,2 et une longueur $d$ de la darse de 0,585 fois la longueur d'onde.

L'agitation dans la darse est alors égale à deux fois l'amplitude du clapotis parfait. Le mouvement de l'eau à l'entrée donne lieu à deux tourbillons verticaux élémentaires se formant alternativement de part et d'autre de la passe. Ils sont dus à la composante normale de la vitesse et relèvent des phénoménes du second ordre. Ces tourbillons dissipent une énergie importante qu'il nous est possible de met- 

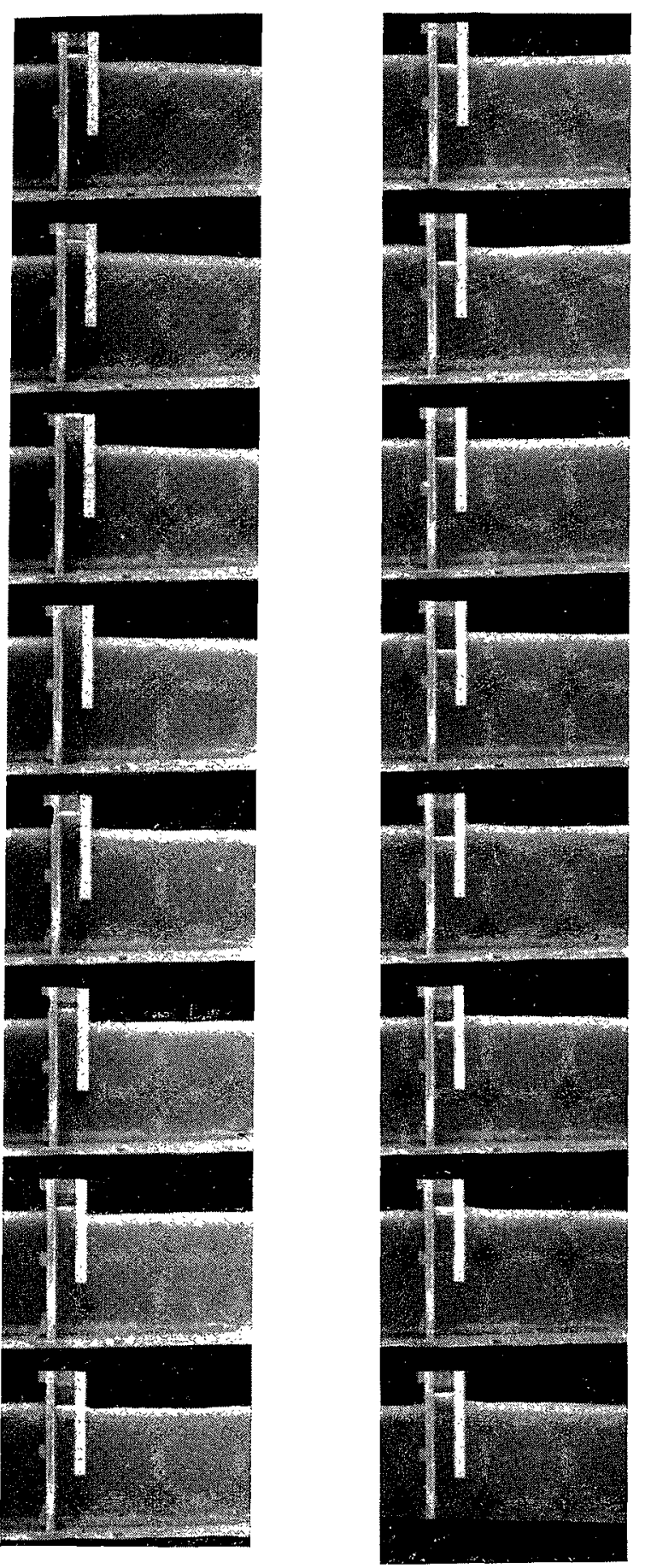

Fic. 10. - Agitation dans un resonateur f'erme par une vanne:

1) Resonance it $\hat{\beta} / 2 \times \mathrm{L}_{2} / 2 \pi$ : leau monle et descend avec une amplitude supérieure à celle du large. La surface de leau entre les deux obstacles reste horizontale.

tre en évidence en disposant deux tôles profilées à l'extrémité des deux murettes limilant la passe d'entrée. L'agitation dans la darse passe alors de quatre fois l'amplitude de la houle incidente ì 4,8 .

\section{d) Fermeture par massifs submergés.}

Des obstacles plans, plus ou moins profilés, entièrement submergés, ont aussi été essayés.
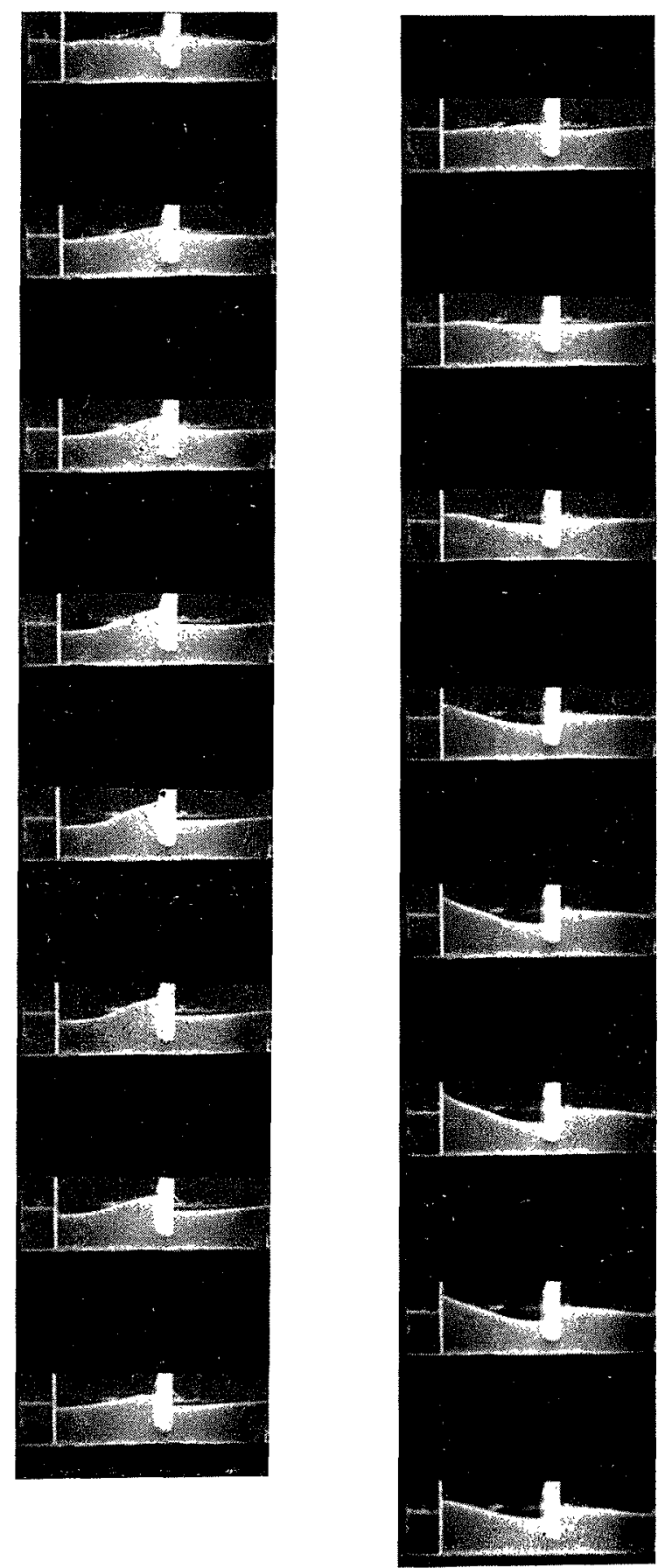

\author{
b) Résonance a $\mathrm{L} / 2+\hat{\beta} / 2 . \mathrm{L} / 2 \pi$ : \\ lamplitude dams lat darse \\ est tres supérieure à l'amplitude du large.
}

Ils n'ont fait que confirmer la théric générale indépendante de la forme exacle de l'obstacle.

Pour ces essais, l'influence de frottement à l'entrée (sur toule la largeur du canal) n'a pas permis d'obtenir une amplification superieure à 5 .

Il peut arriver que pour un obstacle de hateur égale à la profondeur de l'eau dans la darse, done fermant entierement la darse, une résonance 
$1 / 2$ onde très marquée se traduise au droit de l'obstacle par un découvrement périodique de la crête (fig. 11).

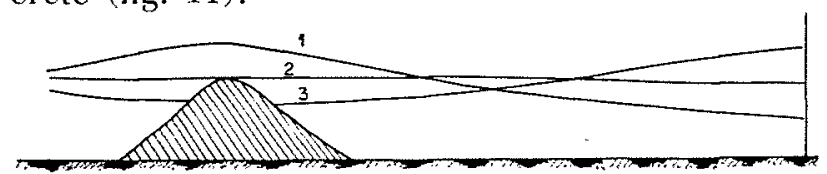

Fia. 11. - Formes particulières de l'agitation. Excitation de la résonance par submersion. gueur d'onde et provoquent, de part et d'autre de ia crête de l'obstacle, un déferlement périodique particulièrement marqué dans le sens darse-large. Ce phénomène permet de voir à lui seul que l'amplitude dans la darse est supérieure à celle du large. Ces phénomènes ont déjà été observés lors des études sur les digues à prédéferlement.

La figure 12 montre la périodicité avec la-

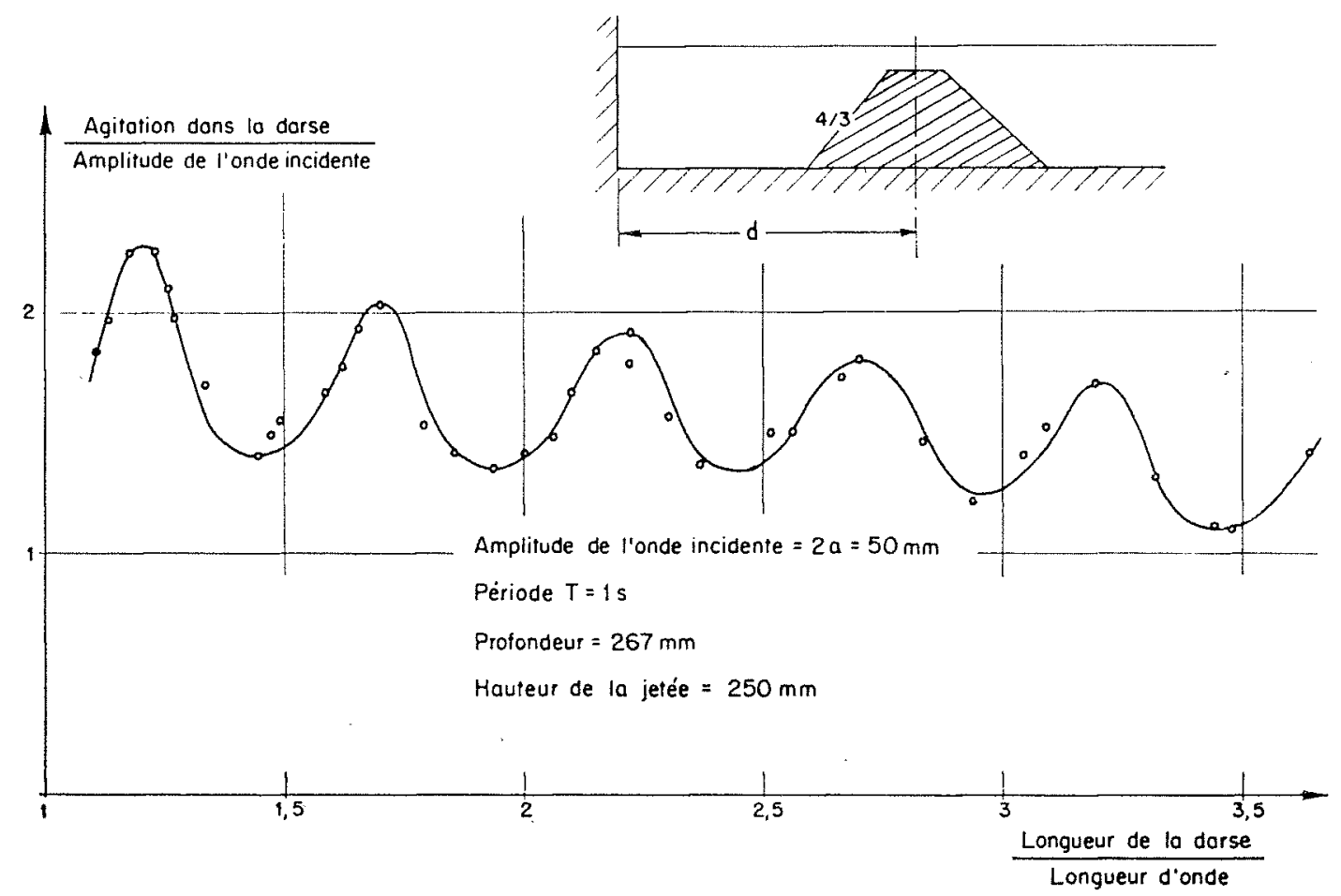

Fif. 12. - Périodicité de la résonance. Les forces excitatrices restant constantes et les forces de frottement augmentant avec le volume de la darse, l'effet de l'amortissement est très net.

D'autres obstacles de moindre hauteur donnent le maximum d'agitation pour une longueur de darse légèrement supérieure à la $1 / 2$ lon- quelle se reproduit le phénomène de résonance et l'amortissement dû aux frottements à l'intérieur de la darse.

\section{c. - DARSE SUR-OUVERTE}

\section{I. - Approfondissement :}

Si l'obstacle localisé est du type « approfondissement $\gg$ (fig. 13), des considérations théoriques analogues aux précédentes, basées sur la conservation de l'énergie d'une part, et la continuilé d'autre part, conduisent à une incompatibilite.
Les essais ont été effectués dans les conditions suivantes : un banc de bois fixé au fond du canal et ayant la largeur de ce dernier provoque un changement brusque de profondeur. Une planche verticale s'enfonçant jusqu'au niveau supérieur de ce bane est fixée à une distance $d$ de son extrémité.

Ceci correspond pratiquement au cas d'une darse peu profonde s'ouvrant sur la pleine mer 


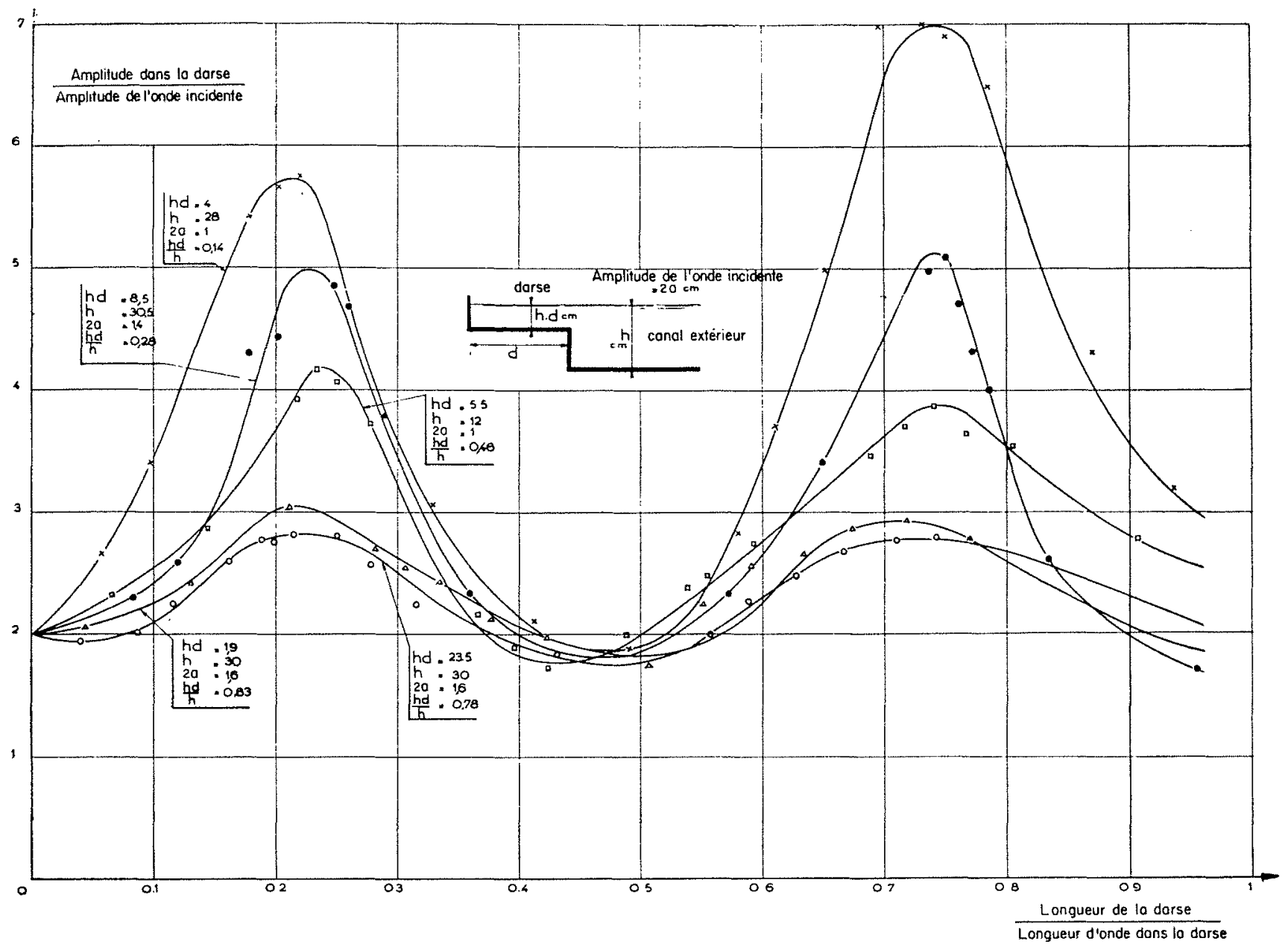

Fig. 13. - Courbes expérimentales donnant la valeur de l'agitation dans une darse sur-ouverte limitée par un approfondissement.

ou, plus généralement, au cas d'un plateau continental donnant sur les grands fonds marins.

Les essais faits dans des conditions expérimentales assez différentes en ce qui concerne l'amplitude de la houle incidente et la profondeur $\mathrm{du}$ canal, montrent cependant très nettement que la résonance a lieu pour des longueurs de darse très voisines mais jamais supérieures à $[k(\mathrm{~L} / 2)+(\mathrm{L} / 4)]$ (fig. 13). Ces courbes montrent, de plus, que l'amplification crôt quand le rapport de la profondeur dans la darse à la profondeur du large décroît. Les essais ont montré que la valeur du minimum d'agitation était voisine de deux fois l'amplitude de l'onde incidente, c'est-à-dire celle du clapotis total, et que la résonance était du type quart d'onde.

\section{II. - Elargissement}

Considérons maintenant une darse pleinement ouverte sur une baie de largeur $b$ (fig. 14). Les caractéristiques de la houle incidente sont les suivantes :

$$
\begin{aligned}
& \text { - Période........ T }=2 \mathrm{~s} \\
& \text { - Profondeur...... } h=20 \mathrm{~cm}
\end{aligned}
$$

Les résultats de ces essais sont consignés par les courbes (14) donnant la valeur de l'agitation rapportée à l'anplitude de la houle incidente en fonction de la longueur relative de la darse. Ces courbes sont tracées pour différentes largeurs de la baie. Elles mettent en évidence que :

1" L'agitation dans une darse ouverte sur un plan d'eau n'est jamais inféricure à l'amplitude du clapolis total;

$2^{\circ}$ Les chances de résonance restent les mêmes quel que soit le rapport de la largeur de la baie à la largeur de la darse; ceci est une différence essentielle entre les conditions de résonance des darses sur-ouvertes et sous-ouvertes ou les chances de résonance diminuent avec la fermeture; 
$3^{\circ}$ La résonance croît avec le rapport de la largeur de la baie à la largeur de la darse et tend asymptotiquement vers une limite conditionnée par les frottements; $4^{\circ}$ Le type de côte accore ou plate avoisinant l'entrée de la darse n'a pratiquement pas d'influence sur les conditions de résonance.

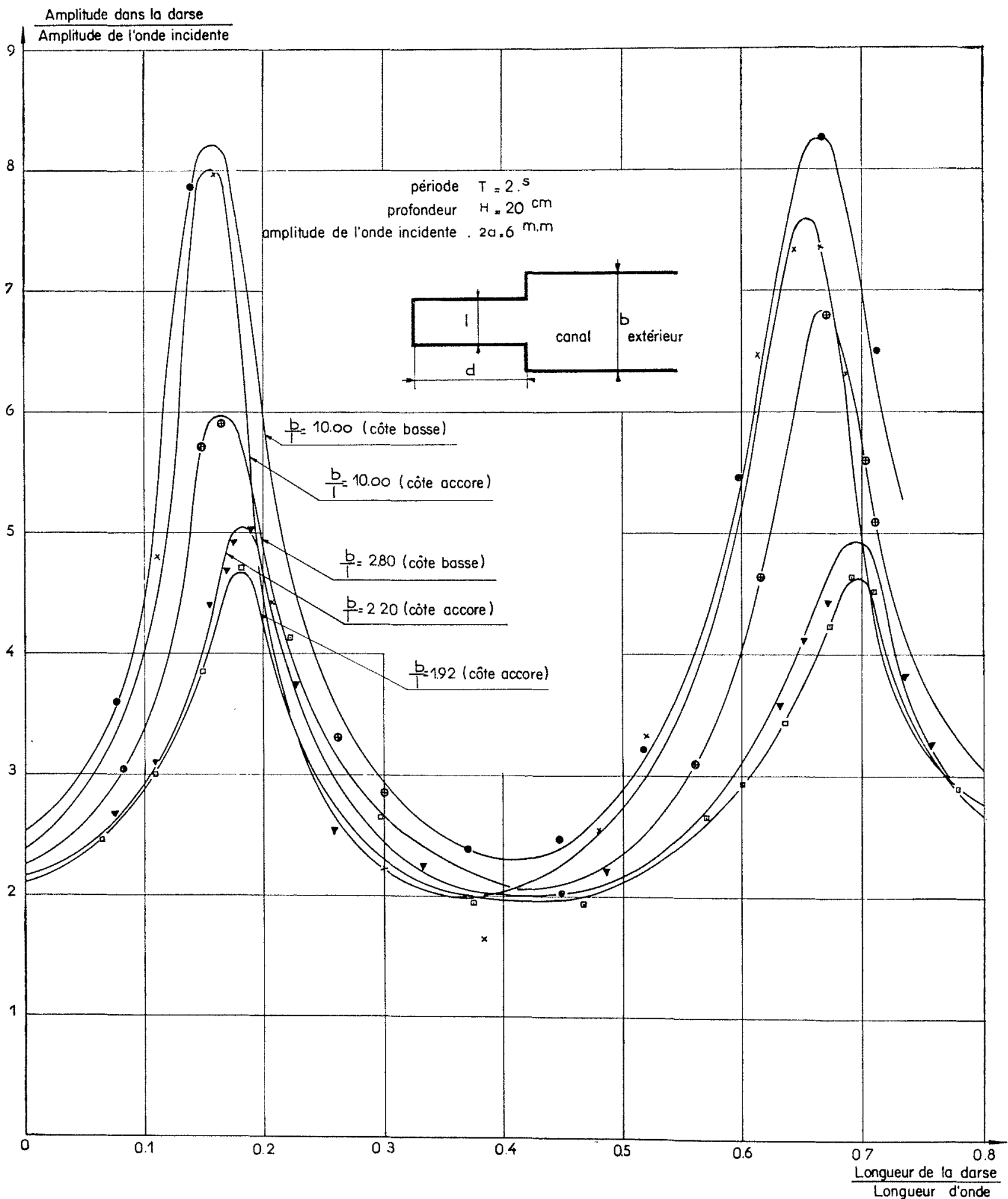

Frc. 14. - Courbes expérimentales donnant la valeur de l'agitation dans une darse sur-ouverte limitée par un élargissement. 
L'influence du clapotis de résonance dans la darse se traduit par un gaufrage radial très accentué, centré sur l'entrée de la darse et résultant de la superposition de la houle incidente

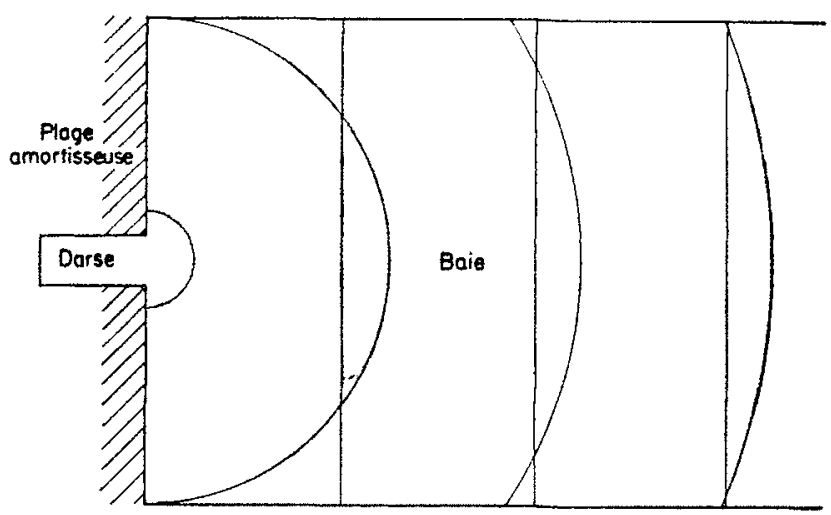

FiG. 15. - Gaufrage radial observé lors de la résonance.

et de la houle réfléchie par la darse seule (fig. 15). Ce phénomène, moins visible dans le cas d'une côte accore, persiste cependant à la résonance.

Pour déterminer les périodes de résonance d'une darse de ce type, Neumann [10] a proposé la formule donnée par BoSANQUET pour les tuyaux sonores, le coefficient expérimental étant alors de 0,346. Cette formule s'exprime sous la forme :

$$
d=\frac{\mathrm{L}}{4}-0,346(l+h)
$$

avec :

$d$ : longueur de la darse;

$\mathrm{L}$ : Iongueur d'onde locale;

$l$ et $h$ : désignent respectivement la largeur et la profondeur de la darse à l'entrée ( $h$ peut être en général négligé devant $l$ ).

Nos essais ont montré que le coefficient expérimental était plutôt égal à 0,4 pour ce type de darse. De plus, on peut généraliser son application en introduisant le facteur $2 k+1, k$ étant un entier quelconque :

$$
d=(2 k+1) \frac{L}{4}-0,4 l
$$

\section{D. - DARSE LIMITEE PAR UN OBSTACLE COMPLEXE}

Le cas d'une darse relativement fermée et donnant sur un bassin d'une largeur et d'une profondeur différentes correspond pratiquement au cas le plus fréquent des ports protégés par une digue en enrochements. Il est alors difficile de préciser si la darse est limitée par un obstacle sur-ouvert ou sous-ouvert.

Quelques essais ont porté sur les conditions de résonance d'une darse limitée par un obstacle complexe formé simultanément d'un rétrécissement et d'un ćlargissement ou d'un approfondissement brusque. Un certain nombre de résultats (fig. 16) sont comparés aux résultats obtenus avec les obstacles simples composants.

Nous avons vu que l'allure des courbes de résonance caractérise nettement l'obstacle sur-ouvert de l'obstacle sous-ouvert. La courbe de résonance obtenue avec l'obstacle complexe a une allure "moyenne » se rapprochant de l'une ou l'autre courbe sujvant que le caractère sur-ouvert ou sous-ouvert de l'obstacle complexe domine. Ce caractère « moyen», difficile à définir avec précision sans quelque théorie plus poussée et des essais plus nombreux, semble vrai en amplitude et en période.

Il est done possible, à partir des courbes de résonance obtenues avec les obstacles simples composants, de tracer approximativement la courbe de résonance de la darse limilée par l'obstacle complexe en remarquant que l'obstacle sous-ouvert semble avoir une influence plus importante sur les conditions de résonance que l'obstacle du type approfondissement, ou élargissement.

Dans le tableau ci-dessous, nous comparons les valeurs des maxima d'agitation et des longueurs de darses correspondantes, obtenues avec des obstacles complexes, et avec les obstacles simples composants.

Nous avons posé :

$d_{f}=k \frac{\mathrm{L}}{2}+\Delta_{f} \quad$ la longueur de la darse sous-

$$
\left(0<\Delta_{f}<\frac{\mathrm{L}}{8}\right)
$$

$d_{0}=k \frac{\mathrm{L}}{2}+\Delta_{i}$ la longueur de la darse surouverte assurant la résonance

$$
\left(\frac{\mathrm{L}}{8}<\Delta_{0}<\frac{\mathrm{L}}{4}\right)
$$
$d=k \frac{\mathrm{L}}{2}+\Delta \quad \begin{aligned} & \text { la longueur de la darse limitée } \\ & \text { par un obstacle complexe as- }\end{aligned}$ surant la résonance.

$A_{f}, A_{0}$ et $A g$ désignant les valeurs des maxima d'agitation correspondants. 
0

Amplitude dans la darse

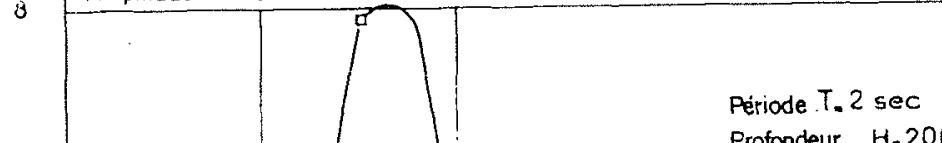

7

2

0

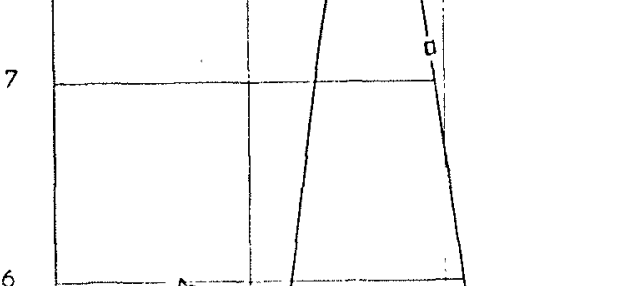

6
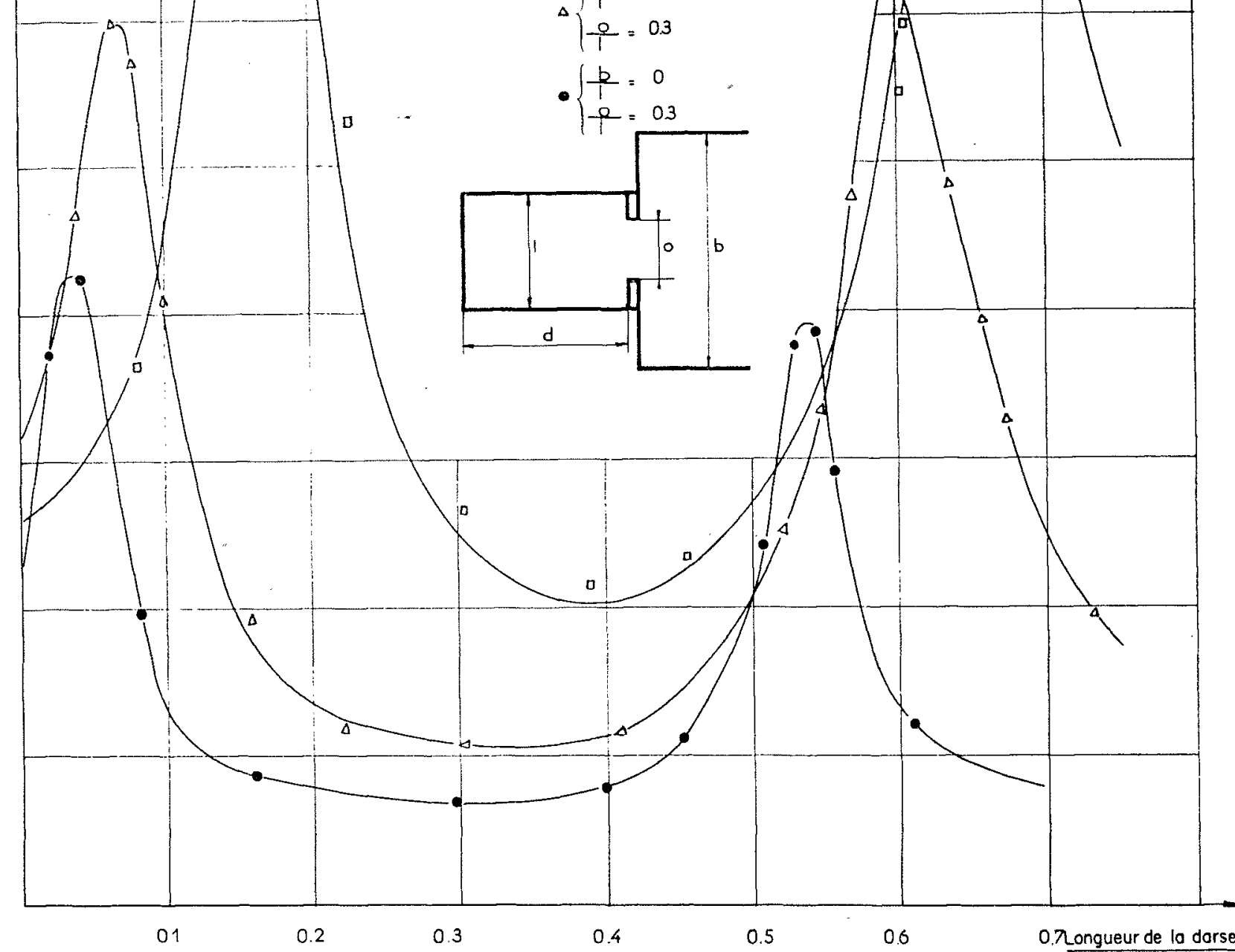

Fic. 16. - Comparaison des courbes d'agitation des darses limites

par un obstacle complexe et de darses limitées par des obstacles simples composants.

\begin{tabular}{|c|c|c|c|c|c|c|c|c|c|}
\hline \multirow{3}{*}{ Obstacle sous ouv } & $0 / l$ & 0,3 & 0,425 & 0,3 & 0,425 & $h^{\prime} / h$ & 0,666 & 0,333 & 0,111 \\
\hline & $\Delta_{f}$ & 0,055 & 0,065 & 0,055 & 0,065 & $\Delta_{f}$ & 0,06 & 0,02 & 0,01 \\
\hline & $A_{f} / 2 a$ & 4 & 3,2 & 4 & 3,2 & $A_{f}$ & 4 & 6,3 & 2 \\
\hline \multirow{3}{*}{ Obstacle sur ouvert....... } & $b / l$ & 10 & 10 & 10 & 10 & $h / h b$ & 0,3 & 0,3 & 0,3 \\
\hline & $\Delta_{n}$ & 0,16 & $\quad 0,16$ & 0,17 & 0,17 & $\Delta_{0}$ & 0,23 & 0,23 & 0,23 \\
\hline & $\mathrm{Ao} / 2 a$ & 8 & 18 & 8,3 & $! 8,3$ & $\mathrm{~A}_{1} / 2 a$ & 5 & 5 & 5 \\
\hline \multirow{2}{*}{ Obstacle complexe......... } & $\Delta$ & 0,06 & 0,08 & 0,07 & 0,09 & $\Delta$ & 0,22 & 0,18 & 0,10 \\
\hline & $\mathrm{A} g|2 a|$ & 5,6 & $\begin{array}{l}6,3 \\
\mid \quad 6\end{array}$ & i 5,8 & 16,8 & $\mathrm{~A} g / 2 a$ & 4,6 & 3,3 & 3,3 \\
\hline
\end{tabular}

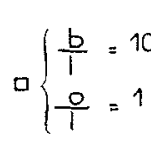

$\Delta\left\{\begin{array}{l}\frac{b}{1}=10 \\ -\underline{p}=0.3\end{array}\right.$

$\left\{\begin{array}{l}b \\ 1\end{array}=0\right.$

$\underline{e}=0.3$
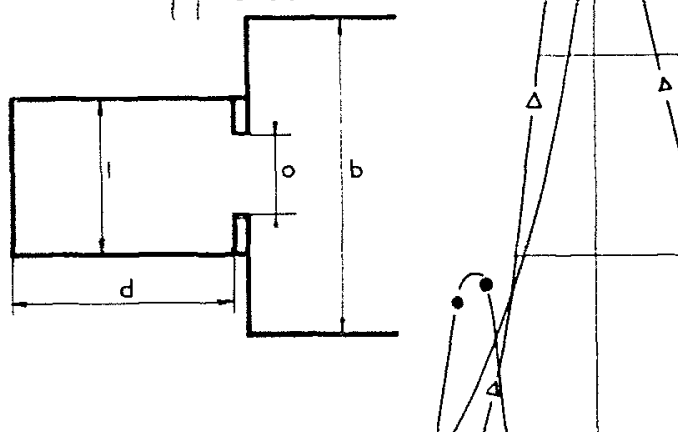
Ce petit nombre de résultats ne permet pas d'exprimer des lois simples avec certitude. Lobstacle complexe est, en effet, plus ou moins localisé et toute possibilité d'accumulation d'eau dans ses limites donne en général priorité à l'obslacle simple le plus près du fond de la darse. Mais cette derniere remarque comporte des exceptions. C'est ainsi que les périodes de résonance de la darse figurée en 17 seraient principalement caractérisées par l'obstacle du týpe obstruction qui se trouve pourtant le plus éloiżné du fond de la darse.

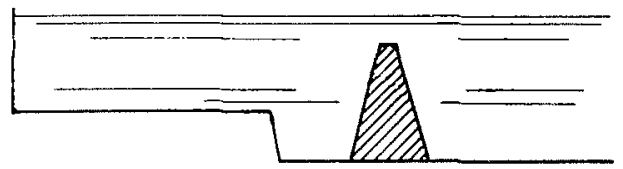

FiG. 17. - L'effet de l'approfondissement sur les lois de résonance est ici moins important que l'effet de l'obstruction.
Cependant, pour les besoins de la pratique, nous pensons que ces quelques résultats nins permettront de donner certaines rigles que nous qualifierons de provisoires. Elles déterminent approximativement les périodes de résonance de n'importe quels bassins où se produisent des mouvements considérés comme bidimensionnels.

Sous ces réserves, les périodes de résonance d'une darse limitéc par un obstacle complexe pourront être données par les formules empiriques suivantes, basées sur la théorie des obstacles simples composantes :

$$
\Delta=\frac{\Delta_{0}+\Delta_{i}}{2}
$$

avec :

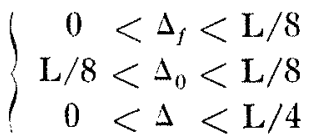

\section{E. - DARSES EN SÉRIE}

Nous examinons ici le cas de darses en série, dans lesquelles pervent se produire des rćsonances en cascade, Ce cas est évidemment rare en pratique, mais présente un intérêt théorique certain, illustrant et vérifiant la méthode de ealcul.

\section{I. - Théorie}

Les formules donnant les valeurs de l'agitation deviennent vite excessivement complexes, mais restent calculables, au moins dans le cas de resonance maxiuum.

Supposons la darse I en résonance (fig. 18). Sa longueur est, comme nous l'avons vu:

$$
d_{1}=\left(J_{1} \pi+\hat{\beta} / 2\right) \frac{\mathrm{L}}{2 \pi}
$$

Par suite du déphasage de la loule lors de son passage sur l'obstacle 1 , les ventres du clapotis extérieur à la darse se frouvent à une dislance de 0 égale à :

$$
\left(k_{1} \pi+\hat{\alpha}\right) \frac{\mathrm{I}}{2 \pi}
$$

Tout se passe done comme si la houle se réfléchissait sur un plan $1^{\prime}$ distant de 0 de cette valeur. De plus, l'agitation dans la darse I est plus grande que l'amplitude extérieure incidente $A_{1}$ dans un rapport déterminé.

Si maintenant, à partir du plan précédem- ment délerminé 1 , on place un deuxieme obstacle identique à une distance 2-1' égale à :

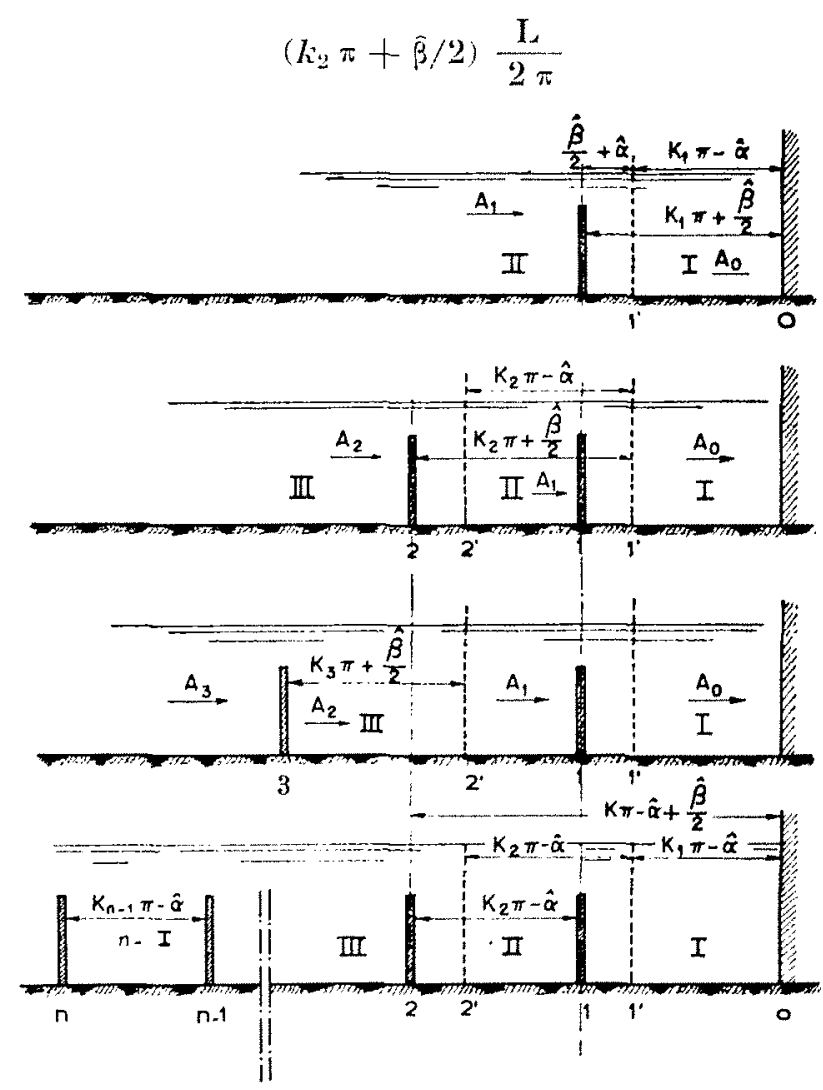

Fic. 18. - Darses en seric. Dans les cas les plus défavorables, l'agitation peut aller en augmentant de darse co darse à mesure que l'on s'éloigne du large. 
on créera une pseudo-darse II, clle-nlême en résonance, où l'amplitude de l'agitalion sera supéricure à l'amplitude incidente extérieure $A_{2}$. La valeur de $A_{1}$ sera donc supérieure à $A_{2}$ par suite de la résonance dans la pseudo-darse 2-1', ou plus exactement dans la darse 2-1. La valeur de l'amplitude de l'agitation ira donc en croissant dans le sens III, II, I.

La distance 2-1 est alors de :

$$
\left(k_{\mathrm{g}} \pi+\bar{\alpha}\right) \frac{\mathrm{L}}{2 \pi}
$$

La houle se réfléchit encore sur le mur $O$, mais les obstacles 1 et 2 introduisent un déphasage $2 \hat{x}$. Les ventres du clapotis au large de l'obstacle 2 seront donc à une distance du mur $O$ égale à :

$$
\left[\left(k_{1}+k_{i}\right) \pi+2 \hat{x}\right] \frac{\mathrm{L}}{2 \pi}
$$

Tout se passe donc au large comme si la houle se réfléchissait sur un plan $2^{\prime}$ distant de 0 de cette valeur.

Une troisiène pseudo-darse III sera en résonance si la distance $3-2^{\prime}$ est égale à :

$$
\left(k_{3} \pi+\hat{\beta} / 2\right) \frac{L}{2 \pi}
$$

Le même raisonnement peut être poursuivi

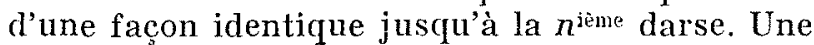
agitation très faible au large peut donc théoriquement provoquer une forte amplilude dans la prenière darse 1 .

Les distances entre les obstacles sont donc respectivement :

enlre 1 et 0 el $:\left(k_{1} \pi+\hat{\beta} / 2\right) \frac{L}{2 \pi}$

entre $n$ el $n-1:\left(k_{n-1} \pi-1-\hat{z}\right) \frac{L}{2 \pi}$

(n étant $>2$ ).

Il est possible de connaitre le déphasage entre $\widehat{A}_{n}$ et $\widehat{\mathrm{D}}_{n-1}$. Tous calculs faits, on lrouve :

$$
\widehat{\mathrm{A}}_{n-} \widehat{\mathrm{D}}_{n-1}=\pi / 2+k \pi
$$

On peul done calculer l'agitation dans chaque darse, compte tenu des condilions aux limites, à savoir l'amplitude de la houle au large.

\section{II. - Vérification expérimentale}

Nous renons de voir qu'il est théoriquement possible d'avoir une agitation croissante de darse en darse à mesure que l'on s'éloigne du large. L'influence des frottements est lelle qu'il est dif- ficile de mettre ce phénomène en évidence. Cependant, avec les obstacles profilés précédemment décrits, il nous a été possible de le vérifier pour deux obstacles successifs dans les conditions suivantes:

- Période : $1 \mathrm{~s}$.

- Amplitude de la houlc : 9,3 $\mathrm{mm}$.

- Profondeur : $13 \mathrm{~cm}$.

- Largeir des passes d'entrée : $6 \mathrm{~cm}$.

- Largeur des darses : $40 \mathrm{~cm}$.

Nous obtenons les résultats suivants :

1) Avec un seul obstacle:

Longueur de la darse $d_{1}=55 \mathrm{~cm}$

Rapport de l'amplitude dans la darse à l'amplitude de la houle au large:

$$
\frac{\mathrm{A} g_{1}}{A_{1}}=3,04
$$

2) Avec deux obsfacles:

Longueur des darses : $d_{1}=55 \mathrm{~cm}$

$$
d_{2}=60 \mathrm{~cm}
$$

$$
\begin{aligned}
& \frac{\mathrm{A} g_{1}}{\mathrm{~A} g_{2}}=1,67 \\
& \frac{\mathrm{A} g_{2}}{\mathrm{~A}_{1}}=2,58 \\
& \frac{\mathrm{A} g_{1}}{\mathrm{~A}_{1}}=4,31
\end{aligned}
$$

Les indices 1 et 2 caractérisent les darses I et II, et Ag est l'amplitude d'agitation au ventre du mouvement.

L'agitation avec deux obstacles est donc 1,44 fois supérieure à l'agrilation avec un seul obstacle. Mais l'agitation dans la darse II est alors 1,18 fois inférieure à l'agitation avec un seul obstacle. Avec trois obstacles successifs, les frottements absorbent un trop fort pourcentage d'énergie pour qu'il soit possible d'amplifier l'agitation dans la darse 1. Cependant, on peut obtenir la même amplitude qu'arec un seul obstacle.

Pratiquement, ce cas de résonance en cascade, nécessitant des conditions peu probables, doit être fort rare. Cependant, les eaux du plateau continental, elles-mêmes en résonance, peuvent induire dans un port une seiche de même période dont l'amplitude sera grande en raison de cetle premicre amplification.

Il faul aussi retenir de celle élude qu'une darse situce au fond d'un port peul, malgré de nombreux étranglements, subir une seiche dans la mesure où les ondes longues ne sont pas absorbées ou réfléchies dans l'avant-port (cas du port de Leixoës). 


\section{F. - INFLUENCE DU FROTTEMENT}

Nous nous attachons maintenant à dégager linfluence des frottements entre les musoirs Centrée et dans la darse sur les conditions de résonance. Ce problème est en eff'et très important pour établir les lois de similitude.

\section{I. - Influence de la cambrure}

Nous savons que le frottement est directement lié à la cambrure de la houle. Plusieurs séries d'essais ont donc été faites dans les limites possibles des générateurs de houle. Les premières, sur une darse rectangulaire fermée par deux musoirs profilés, ont été effectuées dans les conditions suivantes :

1. - Période $\mathrm{T}=2 \mathrm{~s}$.

Profondeur $h=143 \mathrm{~cm}$.

Ouverture relative de la darse $o / l=0,15$.

Longueur relative de la darse $d / L=0,54$.

2. - Période $\mathrm{T}=1 \mathrm{~s}$.

Profondeur $h=133 \mathrm{~mm}$.

Ouverture relative de la darse $=0,125$.

Longueur relative de la darse $=0,53$.

Les résultats obtenus rapportés sur les courbes 19 mettent en évidence pour ces essais une
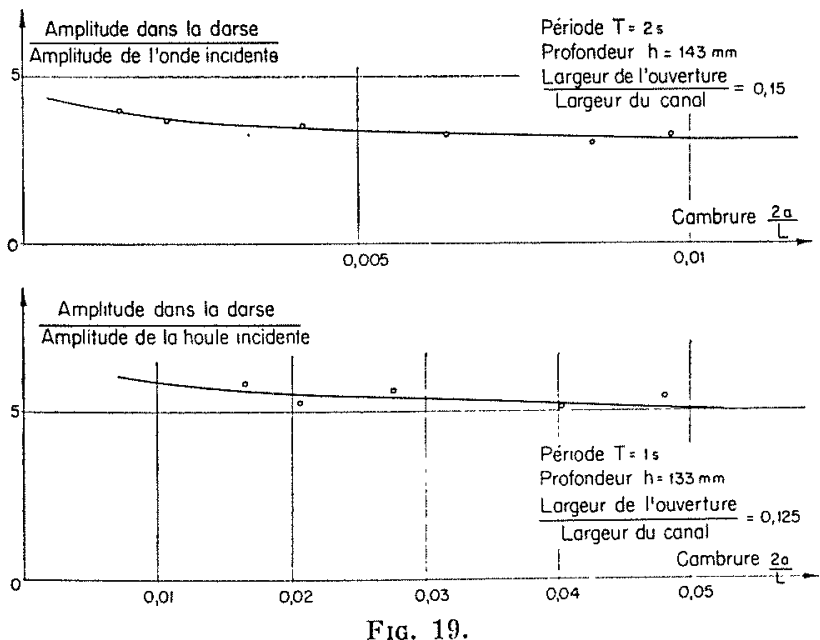

Influence de la cambrure de l'onde incidente.

très légère diminution de l'amplification quand la cambrure croit.

Une autre série de mesures a été faile sur une darse fermée par une vanne plate de $2,5 \mathrm{~cm}$ d'épaisseur. Les conditions d'essais sont alors les suivantes :

$$
\begin{array}{ll}
\text { Période de la houle.... } & \mathrm{T}=1 \text { seconde } \\
\text { Profondeur du canal... } & h=32 \mathrm{~cm}
\end{array}
$$

I.es résultats sont consignés sur les courbes 20 donnant, d'une part la valeur de l'amplitude dans la darse, d'autre part l'amplification de l'agitation en fonction de la cambrure de la houle incidente. On constate que l'agitation atteint asymptotiquement une valeur constante. A partir d'une valeur de la cambrure de la houle incidente do 0,025 , il se produit dans la darse un déferlement à une distance de la vanne, voisine de $(\hat{\beta} / 2) \times(\mathrm{L} / 2 \pi)$. Le déferlement ne peul être olservé que du côté de la vanne, et absorbe l'énergie supplémentaire que fournit la houle quand sa cambrure croît.

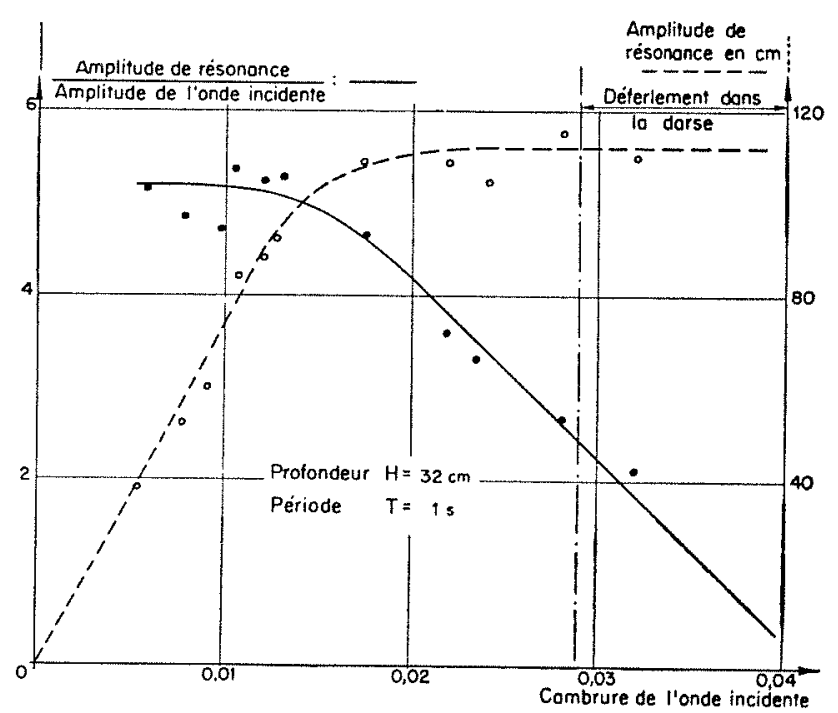

Fici. 20. - Influence de la cambrure de l'onde incidente. Le coefficient d'amplification à la résonance est pratj.quement indépendant de l'amplitude de l'onde incidente tant que n'apparait pas dans la darse une turbulence notable.

Ceci est, d'ailleurs, facilement explicable. L'amplification aurait en effet été constante pour des pertes de charge dues à des frottements proporlionnels aux vitesses (froltements visqueux). Du fait de la turbulence, les frotlements croissent plus vite que suivant celte loi linéaire. Ce point est tres important, car la cambrure des ondes de seiches dans la nature est extrêmement faible. Par consciuent, les ondes sur modele seront com- 
parativement beaucoup plus amorties que dans la nature à la fois par effet d'échelle et parce que l'on y utilise le plus souvent des cambrures fortement exagérées. Cette question est d'ailleurs exposée dans un article sur la similitude des modèles réduits de seiches [2] [4].

\section{II. - Influence de la perte de charge entre les musoirs d'entrée de la rugosité de la darse}

La théorie précédente établie en fluide parfait donne les valeurs des périodes de résonance en fonction de l'angle $\widehat{\beta}$ caractérisant le déphasage de la houle à la réflexion. Une modification de la valeur de cet angle par suite de frottement $\left(x^{2}+\beta^{2}<1\right)$ peut donc modifier les périodes de résonance. Nous avons vu que la longueur de la darse assurant la résonance est :

$$
d=k \frac{\mathrm{L}}{2}+\frac{\hat{\beta}}{2} \times \frac{\mathrm{L}}{2 \pi}
$$

En particulier, lorsque $k$ est nul, toute modification de $\widehat{\beta}$ se traduit, d'après la formule :

$$
\mathbf{T}=\frac{2 \pi}{\beta} \times \frac{2 d}{\sqrt{g h}}
$$

par une modification sensible de la période de résonance.

(Ce résultat peut être rapproché du fait que la période réelle d'oscillation libre d'un bassin fermé est supérieure à celle calculée par la théorie en fluide parfait.)

Dans les essais suivants, nous cherchons donc à dégager l'influence des frottements existant d'une part entre les musoirs d'entrée, et d'autre part dans la darse elle-même. Nous avons placé entre les musoirs profilés limitant la darse un caisson de grillages rempli de copeaux d'aluminium. Ce procédé nous permet d'étudier l'influence de la perte d'énergie à l'entrée sur la période de résonance. Puis, garnissant successivement la darse d'une et deux épaisseurs de grillages ondulés de $16 \mathrm{~mm}$ de maille, il nous a été possible de chiffrer l'importance de la perte par frottement dans la darse sur la période de résonance. Les résultats sont consignés dans le tableau ci-dessous :

Nous nous sommes placés volontairement dans des conditions d'extrême rugosité afin de chiffrer la limite supérieure des effets de frottements. Ces essais ont été effectués avec une longueur de darse égale à $(L / 2)+(\hat{\beta} / 2) \times(L / 2 \pi)$, c'est-à-dire pour $k=1$. La variation relative de la période de résonance due à la perte d'énergie à l'entrée n'est, dans le cas étudié, que de $2 \%$. En pratique, elle ne peul être importante que lorsque $k$ est nul (cas des résonances à $(\hat{\beta} / 2) \times(L / 2 \pi)$. Ce dernier cas sera, par exemple, celui d'une darse de dimensions assez réduites, soumise à des ondes suffisamment longues, ou encore d'une baie ou grande darse soumise à l'action d'ondes très longues, telles que des ondes de marée.

En conclusion, hormis quelques cas particuliers où $k$ est nul, il ne sera pas nécessaire de tenir compte de l'influence du frotlement sur les périodes de résonance. Ceci simplifie done le problème de la similitude (*).

( ) Par contre, les jetées en enrochements élant particulièrement permeables aux ondes longues, il peut ètre utile de respecter la similitude de celte perméabilité daus la mesure ou celle-ci influence l'agitalion à l'inté-

\begin{tabular}{|c|c|c|c|c|c|c|}
\hline \multirow{2}{*}{$\exists$} & \multicolumn{3}{|c|}{$\begin{aligned} 2 a & =0,44 \mathrm{~mm} \\
h & =17 \mathrm{~cm}\end{aligned}$} & \multicolumn{3}{|c|}{$\begin{aligned} 2 a & =0,74 \mathrm{~mm} \\
h & =17 \mathrm{~cm}\end{aligned}$} \\
\hline & $\mathrm{A} g / 2 a$ & $d / \mathrm{L}_{4}$ & & $\mathrm{~A} g / 2 a$ & $d / \mathrm{L}$ & \\
\hline Sans rugosité.. & 3,95 & 0,542 & & 2,42 & 0,542 & \\
\hline Entrée rtigueuse. & 2,6 & 0,526 & très rugueuse & 1,83 & 0,530 & rugueuse \\
\hline Fond rugueux... & 3,55 & 0,54 & $\begin{array}{l}\text { une épaisseur } \\
\text { de grillage }\end{array}$ & 2,12 & 0,530 & $\begin{array}{l}\text { deux épaisseurs } \\
\text { de grillage }\end{array}$ \\
\hline Entrée et fond rugueux. & 2,6 & 0,522 & & 1,35 & 0,526 & \\
\hline
\end{tabular}
rieur des bassins étudiés [17]. 


\section{G - COMPARAISON ENTRE L'AMPLITUDE DES COMPOSANTES HORIZONTALES DU MOUVEMENT DANS LA DARSE ET DANS LA PASSE D'ENTREE}

L'amplitude du mouvement horizontal de l'eau aux nœuds peut facilement être calculée à partir

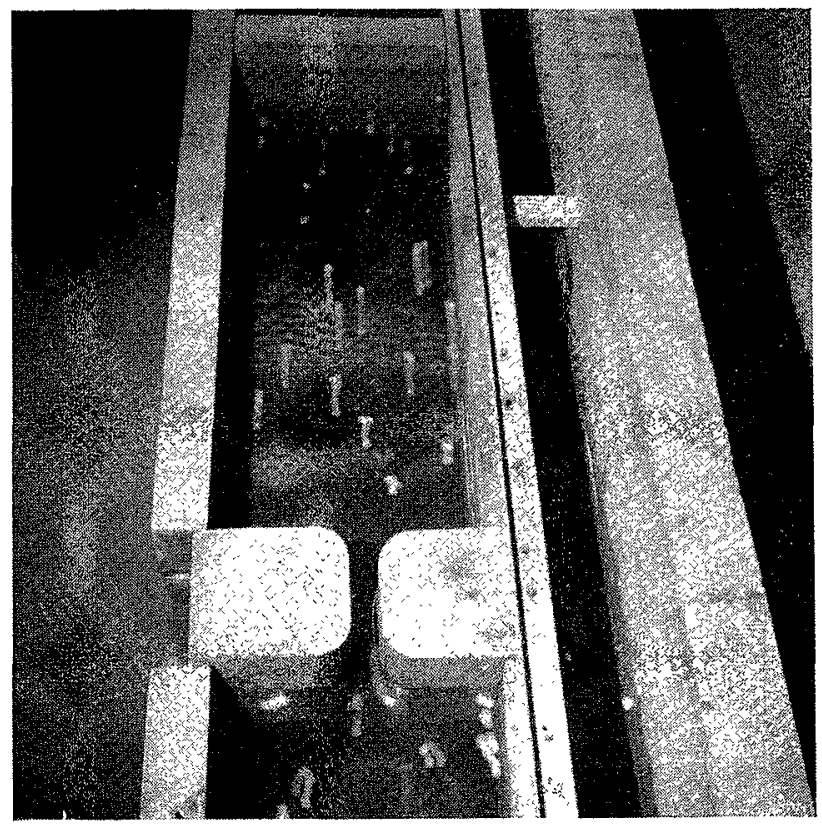

(a)

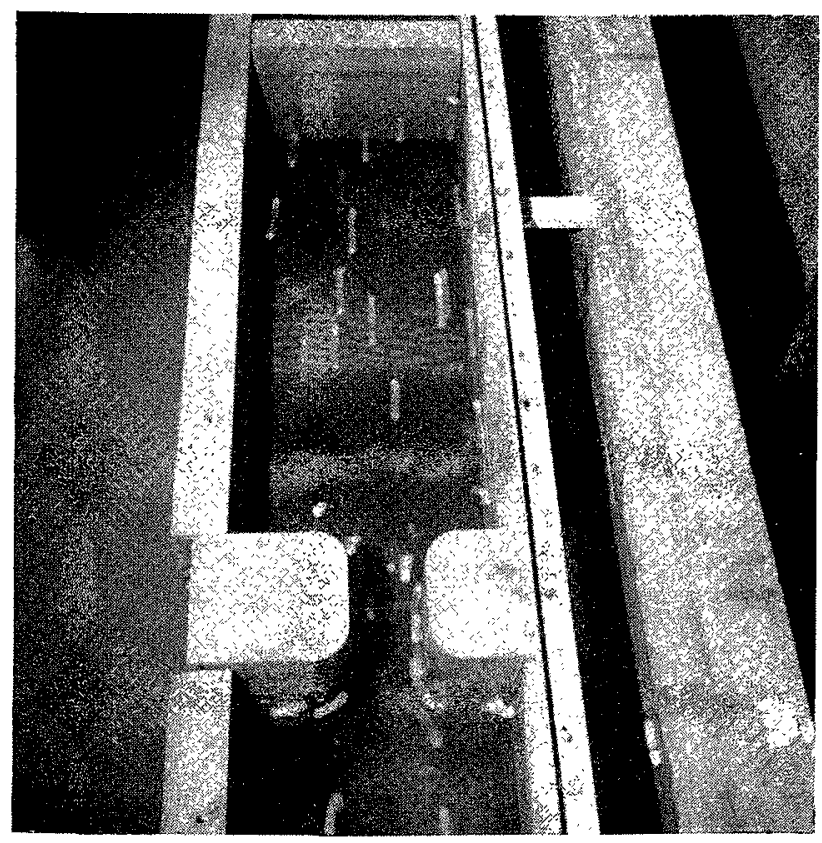

(b) de l'amplitude $\mathrm{A} g$ du mouvement vertical au ventre, par la formule simple:

$$
n=\frac{\mathrm{A} g \cdot \mathrm{T}}{2 \pi} \sqrt{\frac{g}{h}}
$$

$h$ élant la profondeur de la darse et T la période de mouvement.

C'est ainsi que pour $\mathrm{A} g=3 \mathrm{~cm}, \mathrm{~T}=\mathbf{2} \mathrm{s}$, $h==13 \mathrm{~cm}, n$ est égal à $8,68 \mathrm{~cm}$, soit 2,9 fois l'amplitude du mouvement vertical au ventre.

Une série de photographies a permis, au moven de flotteurs, de vérifier grossièrement cette formule (fig. 21). Mais elle mettent surtout en évidence l'importance du mouvement horizontal a la passe d'entrée. Ce mouvement dans la passe d'entrée est dû à la différence de niveau au droit de l'obstacle entre la darse et l'extérieur. Celte différence de niveau provient d'une part de la différence d'amplitude (à la résonance), d'autre part du déphasage précédemment calculé.

Pratiquement, l'amplitude du mouvement horizontal dans la passe d'entrée reste sensiblement la même, quel que soit le degré d'ouverture de la darse. Ceci provient alors du rapprochement vers l'obstacle des nouds du clapotis du large. Pour de fortes cambrures de la houle inci-

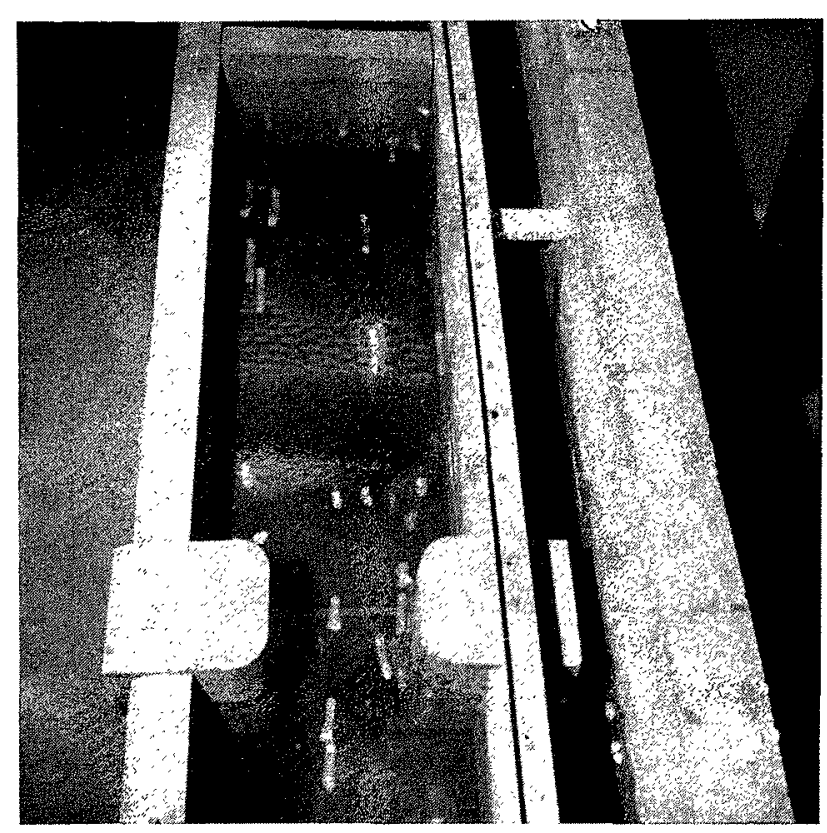

(c)

Frg. 21. - Dans le cas de la résonance $1 / 2$ onde, les courants dans la passe d'entréc sont toujours aussi violents que dans le cas de la résonance 1/4 d'onde. Il ne faut donc pas conclure à une résonance de ce dernier type sur cette unique observation. 
dente, conduisant à une très forte agitation dans la darse, il est possible d'observer dans la passe d'entrée un véritable déferlement périodique.

Il ne faut donc pas conclure, sur la seule observation d'un mouvement violent dans la passe d'entrée, à une résonance $1 / 4$ d'onde. Dans le cas présent, où la darse débouche sur un canal de même largeur et de même profondeur, la résonance quart d'onde ne peut exister.

D'ailleurs, dans le cas d'une résonance $1 / 4$ d'onde obtenue avec une darse sur-ouverte, les amplitudes horizontales au ventre du mouvement et à la passe d'entrée sont évidemment sensiblement égales entre elles.

\section{H. - VÉRIFICATION DE LA THÉORIE SUR LES OBSTACLES [1]}

La mesure des coefficients de transmission $\alpha$ et de réflexion $\beta$ a été faite pour le type d'obstacle composé de deux musoirs en béton (fig. 6).

Les mesures ont été faites à l'enregistreur graphique de houle.. Le coefficient de transmission $\alpha$ peut être obtenu directement en aval de l'ouvrage. La valeur de $\beta$ est donnée à partir des amplitudes aux ventres $A$ et aux nouds $B$ en amont de l'ouvrage par la formule simple :

$$
\beta=\frac{A-B}{A+B}
$$

Les valeurs ainsi trouvées sont reportées sur les courbes 22 et 23 en fonction de la largeur re-

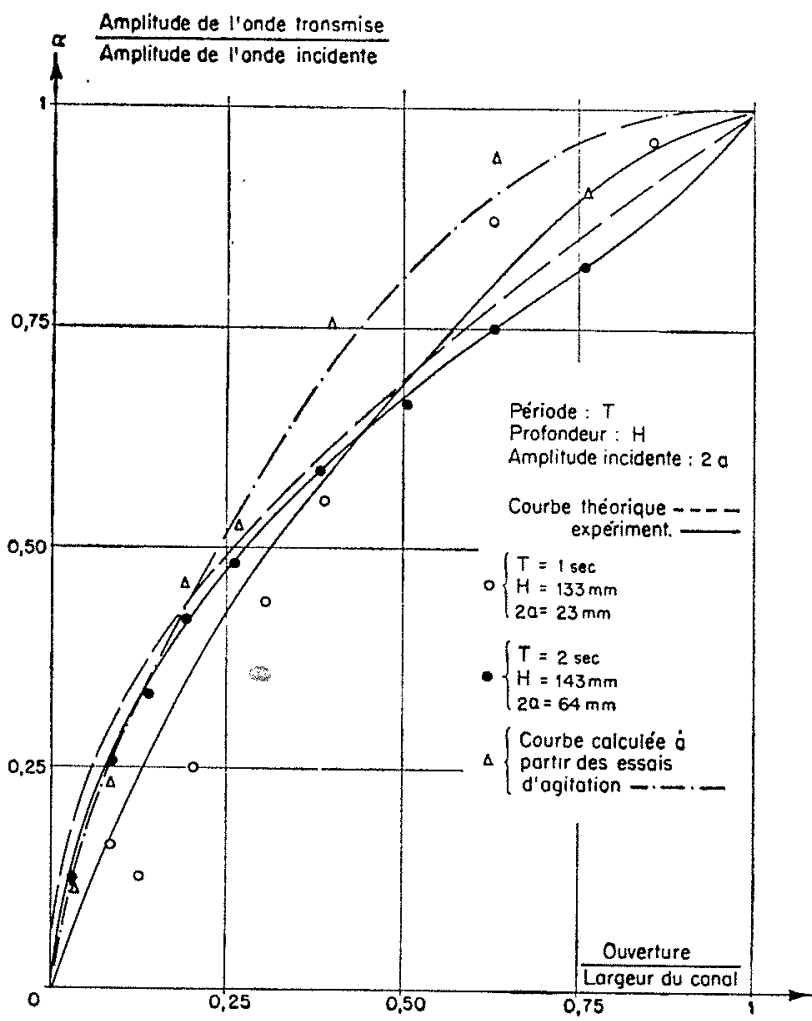

FIG. 22.

Coefficient de transmission $\bar{\alpha}$ d'un obstacle dans un canal.

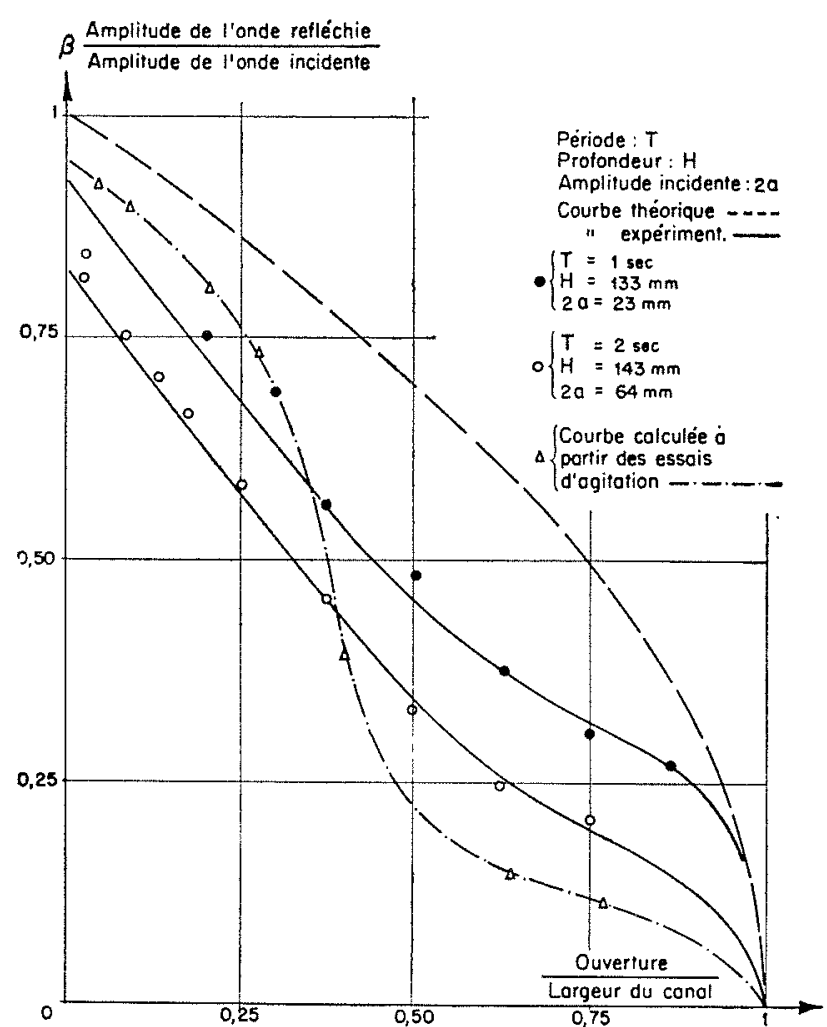

Fig. 23.

Coefficient de réflexion $\beta$ d'un obstacle dans un canal.

lative $o / l$ de la passe. Nous avons tracé sur les mêmes graphiques les courbes donnant $\alpha$ et $\beta$ à partir des relations approximatives :

$$
\begin{aligned}
& \alpha=\sqrt{\frac{o}{l}} \\
& \beta=\sqrt{\frac{l-o}{l}}
\end{aligned}
$$

Ces formules permettent de calculer des courbes théoriques admettant la conservation de l'énergie. D'autre part, celles calculées à partir 
des valeurs $A_{M}$ et $A_{m}$ des agitations maxima et minima obtenues lors des essais d'agitation entre deux obstacles, permettent aussi de déduire les valeurs de $\alpha$ et $\beta$ :

$$
\begin{aligned}
& \alpha=\frac{A_{\mathrm{M}} \times A_{m}}{A_{1}\left(A_{\mathrm{MI}}+A_{m}\right)} \\
& \beta=\frac{A_{\mathrm{ML}}-A_{m}}{A_{\mathrm{M}}+A_{m}}
\end{aligned}
$$

La concordance entre ces différentes expressions de $\alpha$ et $\beta$ est bonne à la transmission, moins bonne à la réflexion. Sans doute faut-il attribuer ces divergences à l'influence des frotlements et au profil complexe des ouvrages pour lesquels la

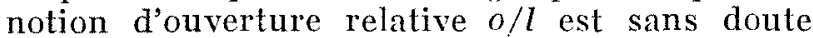
trop simpliste.
La mesure de $\hat{\beta}$ a été faite à partir des distances des nouds (et éventuellement des ventres) du clapotis partiel devant l'obstacle, suivant la formule :

$$
\bar{\beta}=\left(\mathrm{N}-\frac{2 n-1}{4} \mathrm{~L}\right) \frac{4 \pi}{\mathrm{L}}
$$

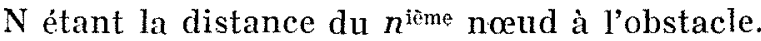

Les résultats ainsi obtenus pour $\mathrm{T}=1$ seconde donnent une valeur de $\bar{\beta}$ nulle par rapport au plan amont de l'ouvrage, quelle que soit la largeur de la passe; mais la «localisation" de l'obstacle est dans ce cas douteuse. Il est probable qu'un obstacle localisé eût vérifié plus sûrement la théorie générale.

\section{I. - DARSE LIMITEE PAR UN OUVRAGE PERMEABLE}

Nous avons vu dans la théorie des obstacles soumis à la houle qu'un ouvrage absorbant de l'énergie pouvait répondre à la condition :

$$
\alpha+\beta=1
$$

(ouvrage purement résistant).

Un massif en enrochements de largeur faible par rapport à la longueur d'onde satisfait sensiblement cette cóndition.

Suivant les mêmes principes que ceux utilisés dans l'étude des darses sous-ouvertes, il nous est possible de déterminer la valeur de l'agitation entre un ouvrage parfaitement réfléchissant et un ouvrage purement résistant. L'application de la méthode des images appliquée à ce cas donne directement la valeur de l'agitation :

$$
\mathrm{A} g=-\frac{2 \bar{r}^{1 / 2}(1-\bar{\beta})}{\sqrt{1+\bar{\beta}^{2}-2 \bar{\beta} \cos 0}} \mathrm{~A}_{1}
$$

D'où les courbes théoriques (24) donnant l'amplitude maximum de l'agitation en fonction de $\bar{\beta}$ (ou de $\bar{\alpha}$ ). Remarquons que lagitation dans la darse est toujours inférieure ou au plus égale à l'agitation au large. Ce dernier cas se produit lorsque la longueur de la darse est égale à un nombre entier de fois la demi-longueur d'onde. Il $\mathrm{y}$ a alors un ventre de mouvement au droit de l'ohstacle perméable mince. Les mouvements ho- rizontaux sont done nuls et l'absorption d'énergie est théoriquement nulle. Les courbes d'agitation présentent cependant des maxima, nous dirons qu'il y a alors : "pseudo-résonance».

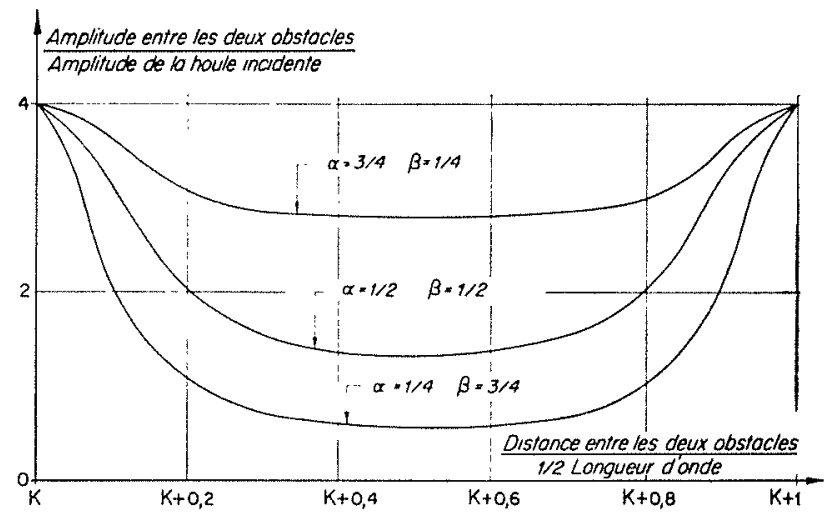

Fig. 24. - Agitation entre un obstacle parfaitement réfléchissant et un obstacle purement résistant.

Des essais effectués dans des conditions analogues aux études précédentes nous ont permis de vérifier cette théorie.

Ces résultats (fig. 25) montrent que le maximum théorique de l'agitation égal à $2 \mathrm{~A}_{1}$ n'est pratiquement jamais atleint. Ceci est principalement dû au fait que l'obstacle perméable ne pou- 


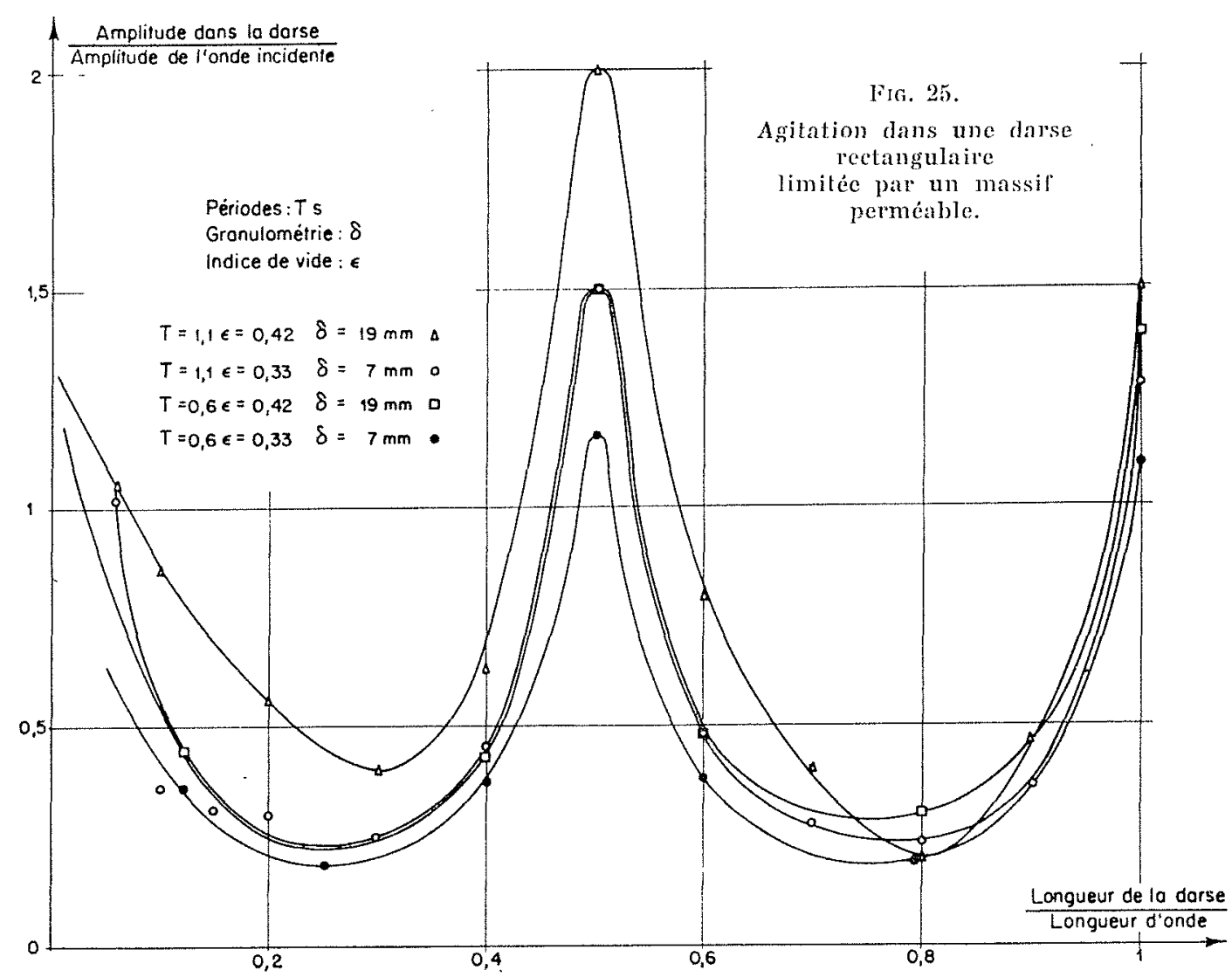

vant avoir une largeur infinimenl petite, il existe toujours des perles d'énergie, dues aux mouvements de l'eau à travers ]'ouvrage. On constate d'ailleurs que le maximum est d'autant plus difficile à atteindre que le rapport de cette largeur à la longueur d'onde $\mathrm{L}$ est important.

\section{J. - ESSAIS DE SYNTHÈSE}

Nous proposons maintenant un certain nombre de lois de résonance ayant pour but de tirer les conclusions générales de l'étude des' seiches dans une darse rectangulaire de profondeur constante. La majorité de ces lois' reste qualitativement valable pour un port de profondeur quelconque et de forme allongée. Elles présentent donc une certaine généralité.

\section{I. - Période de résonance. Degré d'ouverture}

En remplacement des formules antérieures donnant les périodes de résonance d'une darse ouverte :

$$
\mathrm{T}=\frac{4}{2 k+1} \int_{0}^{d} \frac{d x}{\sqrt{g h(x)}}
$$

et d'une darse fermée :

$$
\mathrm{T}=\frac{2}{k+1} \int_{0}^{a} \frac{d x}{\sqrt{g h(x)}}
$$

nous proposons la formule :

$$
\mathrm{T}=\frac{4}{2 k+\mathrm{D}} \int_{0}^{d} \frac{d x}{\sqrt{g h(x)}}
$$

dans laquelle D est le «degré d'ouverture »compris entre 0 et 1. Le degré d'ouverture est pratiquement indépendant de $k$ pour les darses sousouvertes et les darses sur-ouvertes du type approfondissement. Par contre, il est fonction de $k$ (donc de la période) pour les darses sur-ouvertes du type élargissement.

Celle formule implique que dans le cas d'un bassin rectangulaire de profondeur constanle, la résonance ne peul avoir lien que lorsque la darse 
a une longueur $d$ exprimée en longueur d'onde $L$ comprise entre :

$$
k \frac{\mathrm{L}}{2} \text { pour } \mathrm{D}=0
$$

et :

$$
(2 k+1) \frac{\mathrm{L}}{4} \text { pour } \mathrm{D}=1
$$

quel que soit le degré d'ouverture, c'est-à-dire quel que soit le type de l'obstacle à l'entrée.

De plus, les minimums d'agitation ont toujours lieu lorsque la darse a une longueur comprise entre :

$$
(2 k+1) \frac{\mathrm{L}}{4} \text { et }(k+1) \frac{\mathrm{L}}{2}
$$

quel que soit le type de l'obstacle à l'entrée.

a) Dans le cas d'une darse sous-ouverte, le degré d'ouverture est une fonction du rapport de l'ouverture de la darse o sur la largeur de celle-ci $l$, ou du rapport de l'ouverture de la vanne $h^{\prime}$ sur la profondeur dans la darse $h$ (fig. 26). D est alor's compris entre 0, dans le

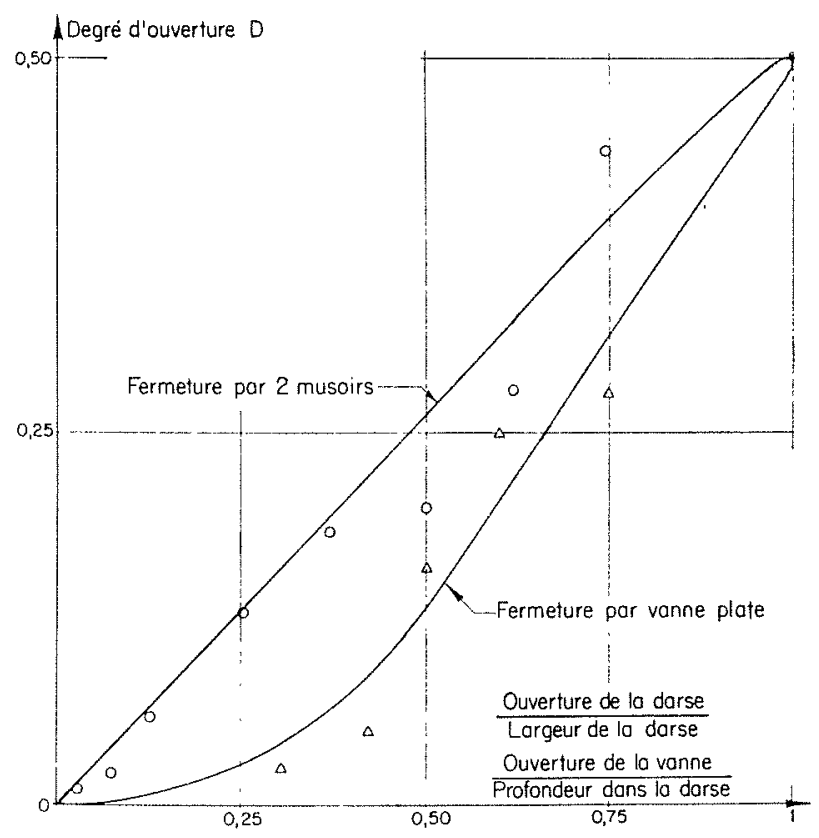

Fis. 26. - Degré d'ouverture d'une darse rectangulaire sous-ouverte. Courbes expérimentales.

cas de lobstruction totale, et, théoriquement, 0,5 dans le cas de l'obstruction nulle.

La résonance a donc lieu pour une darse fermée lorsque sa longueur est comprise entre:

$$
k \frac{\mathrm{L}}{2} \text { lorsque } \mathrm{D}=\mathrm{D}_{f}=0
$$

et une valeur voisine de :

$$
k \frac{\mathrm{L}}{2}+\frac{\mathrm{L}}{8} \text { lorsque } \mathrm{D}=\mathrm{D}_{j}=0,5
$$

b) Dans le cas d'une darse ouverte (type élargissement), $\mathrm{D}=\mathrm{D}_{0}$ est une fonction de $(l+h) / \mathrm{L}$ et de $k$ dans laquelle $l$ et $h$ désignent la largeur et la profondeur de la darse à l'embouchure.

Tous calculs faits, le degré d'ouverture donné par la formule expérimentale de Neumann s’écrit :

$$
\mathrm{D}_{0}=\frac{d-0,2 k \times 0,4(l+h)}{d+0,4(l+h)}
$$

Pratiquement, $D_{0}$ reste, dans ce cas, compris entre 0,5 et 1 .

La résonance a lieu pour une darse ouverte, limitée par un obstacle du type élargissement, lorsque sa longueur est voisine de :

$$
d=(2 k+1) \frac{\mathrm{L}}{4}-0,4(l+h)
$$

c) Dans le cas d'une darse sur-ouverte (type approfondissement), le degré d'ouverture $\mathrm{D}=\mathrm{D}_{0}$ est sensiblement compris entre 0,9 et 1 .

La longueur de résonance de la darse tend alors par valeur inférieure vers :

$$
d=(2 k+1) \frac{\mathrm{L}}{4}
$$

La correction d'embouchure n'est pas supérieure à $0,1 \mathrm{~h}$. L est la longueur d'onde dans la darse ( $\left.{ }^{\star}\right)$.

d) Cas d'une darse limitée par un obstacle localisé complexe décomposable en obstacles simples :

Dans le cas général ò̀ aucun des obstacles simples composants ne prédomine par son caractère ouvert ou fermé, on prendra en toute première approximation :

$$
\mathrm{D}=\frac{\mathrm{D}_{0}+\mathrm{D}_{1}}{2}
$$

(*) Un eas pratique très fréquent est celui figuré en (27) representant une coupe en plan du plateau continental. On peut alors considerer le plateau continental comme un bassin à pente constante débouchant sur un grand fond. Les périodes de résonance sont données par l'expression :

$$
\mathrm{T}=\frac{\bullet \sqrt{g h_{0}}}{4 \pi d}
$$

dans laquelle $d$ et ho désignent respectivement la longueur et la profondeur maximum du plateau continental, $\varrho$ étant l'une des $(2 n+1)$ fimes racines annulant la fonction .To de Bessel.

$$
Q=2,405 ; 5,520 ; 8,654 \ldots
$$




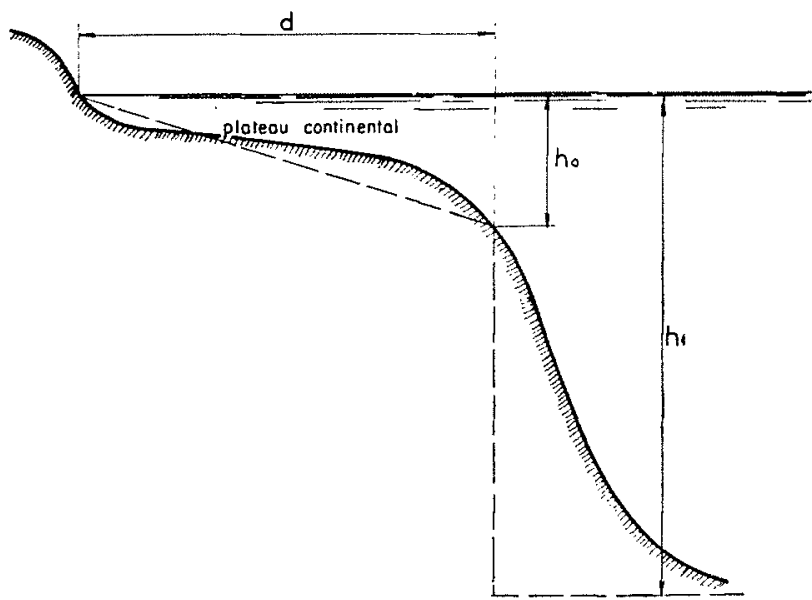

Fıa, 27. - Le plateau continental peut souvent être assimilé à une darse sur-ouverte dont il est facile de calculer les périodes de résonance.

La longueur de résonance de la darse linitée par un obstacle localisé complexe est alors toujours comprise entre :

$$
k \frac{\mathrm{L}}{2}<d<k \frac{\mathrm{L}}{2}+\frac{\mathrm{L}}{4}
$$

c) Cas d'une darse limitée par un ouvrage perméable: les périodes de «pseudo-résonance 》 sont données en faisant :

$$
\mathrm{D}=0
$$

La longueur de «pseudo-résonance» de la darse est toujours :

$$
d=k \quad \frac{\mathrm{L}}{2}
$$

\section{II. - Amplitude de l'agitation}

$1^{\circ}$ Toutes choses égales d'ailleurs, l'amplitude de l'agitation dans une darse est sensiblement proportionnelle à l'amplitude de l'onde incidente. La non-linéarité des termes de frottement turbulent contribue à détruire cette proportionnalité;

$2^{\circ}$ Il suffit d'une énergie incidente extrèmement faible, surtout pour une darse fermée, pour entretenir, lors de la résonance, un phénomène de seiche extrêmement violent. Cet apport d'énergie est alors égal aux pertes par frottement dans la darse;

$3^{\circ}$ Plus une darse a un caractère sous-ouvert, plus l'amplitude de résonance est accentuée, jusqu'à concurrence du frottement particulièrement important entre les musoirs d'entrée de la darse sous-ouverte (fig. 28).

En fluide parfait, si $A_{M}$ est l'amplitude de résonance, $\mathbf{A}_{m}$ l'amplitude minimum de l'agitation obtenue pour une période différente de l'excitation, et 2 a l'amplitude incidente extérieure sup- posée constante, on a, pour une darse sous-ouverte, la relation :

$$
A_{\mathrm{NI}} \times A_{i m}=16 a^{2}
$$

dans le cas pratique le plus fréquent pour les seiches portuaires où $\alpha$ et $\beta$ ne dépendent pas de la Iongueur d'onde.

Le minimum d'agitation d'une darse sur-ouverte n'est pas inférieur à l'amplitude $4 a$ du clapotis pur;

$4^{\circ}$ L'agitation dans une darse sous-ouverte est en moyenne plus faible que l'agitation dans une darse sur-ouverte, car il faut un temps plus long pour mettre en résonance une dar'se sous-ouverte, et la période excitatrice n'est, dons la nature, jamais rigoureusement constante;

$5^{n}$ L'amplitude de résonance des eaux d'une darse limitée par un obsiacle localisé complexe est du même ordre de grandeur que les amplitudes de résonance des darses limitées par les obstacles simples composants, toutes choses égales par ailleurs;
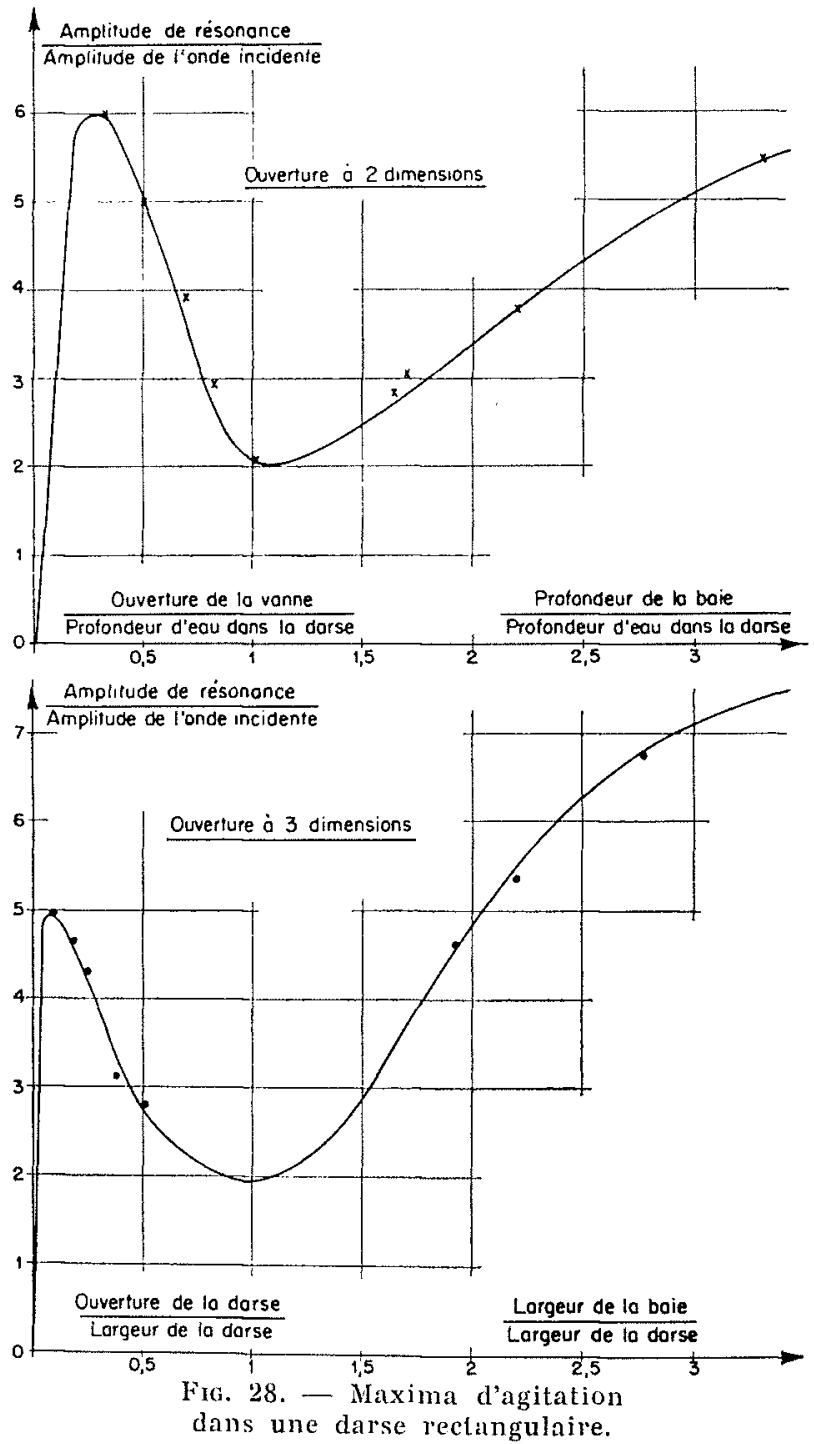
$6^{\circ}$ Dans le cas le plus défavorable, l'amplitude de l'agitation peut aller en augmentant de darse en darse à mesure que l'on s'éloigne géométriquement de l'incidence de la cause excitatrice. En fait, le frottement intervient rapidement pour limiter l'amplification.

Plus fréquemment, une arrière-darse peut roir ses eaux résonner sans qu'aucun mouvement ne soit perceptible dans l'avant-port;

$7^{\circ}$ L'amplitude de «pseudo-résonance »dans une classe limitée par un ouvrage perméable est égale ou inférieure à deux fois l'amplitude de l'onde excitatrice.

\section{III. - Aspect des courbes d'agitation}

$1^{\circ}$ Plus une darse est sous-ouverte, plus la gamme des périodes de résonance est étroite et plus la zone de minimum d'agitation est large, tandis que l'étendue de la gamme des périodes de résonance d'une darse ouverte est indépendante de son degré d'ouverture.
En effet, les courbes de résonance d'une darse sur-ouverte peuvent toutes se déduire les unes des autres par une translation parallèle aux abscisses (fonction de $(l+h) / L$ ) et par une affinité d'axe $\left(2 A_{1}\right)$ fonction de la valeur de l'élargissement ou de l'approfondissement;

$2^{\circ}$ Les courbes donnant l'agitation en fonction de la longueur de darse sont symétriques par rapport à des parallèles aux ordonnées passant par les maximums et les minimums. L'emplacement des minimums est à $=!-\mathrm{L} / 4$ de celui des maximums ;

$3^{\circ}$ La courbe d'agitation d'une darse limitée par un obstacle localisé complexe est une « moyenne» des courbes d'agitation des darses limitées par les obstacles simples composants, compte tenu des formules empiriques antérieures;

$4^{\circ}$ Quelles que soient les conditions de résonance el pour tous types de darse, les courants à l'entrée sont toujours très violents et du mème ordre de grandeur que ceux produits aux nouds du mouvement dans la darse.

\section{CONCLUSION}

La concordance entre la théorie et les résultats expérimentaux met en évidence l'intérêt pratique du calcul des nombres complexes dans l'analyse des mouvements périodiques linéaires à deux dimensions.

Les lois émises contribuent à exprimer la plupart des phénomènes de résonance dans les ports, les baies, ou sur le plateau continental. Elles sont valables quelle que soit la période et s'appliquent aux mouvements provoqués par les houles, les ondes de seiches (Table Bay, Leixöes) ou les ondes de marées (Golfe de Gabès).

La résonance ne peut avoir lieu que lorsque la longueur de la darse exprimée en longueur d'onde est comprise entre :

$$
k \frac{L}{2} \text { et }(2 k+1) \frac{L}{4}
$$

quel que soit le type de passe d'entrée (c'està-dire entre $o$ et $\mathrm{L} / 4$, entre $\mathrm{L} / 2$ et $3 \mathrm{~L} / 4$, etc.).

Il est important de noter, sur le plan pratique, que, dans la nature, les ondes excitatrices n'ont jamais une période rigoureusement constante. Par suite, le degré de sélectivité des courbes d'agitation permet d'établir la probabilité de résonance des darses en fonction de leur degré d'ouverture, compte tenu du nombre d'ondes nécessaires pour provoquer cette résonance, fonction elle aussi du degré d'ouverture. En effet, le
Lemps de mise en résonance de la darse est directement fonction de l'énergie incidente, c'està-dire de la largeur de la passe d'entrée.

Compte tenu du degré de sélectivité des courbes de résonance, on peut donc conclure:

1. Qu'une darse limitée par une entrée très étroite dont la courbe de résonance est tris sélective a relativement peu de chance de résonner. En effet, la résonance s'établit lentement et la période de l'onde excitatrice peut changer avant gue le maximum d'agitation soit atteint. Par contre, la résonance peut être très accentuée lorsque la régularité de l'excilation est exceptionnelle.

2. Au contraire, un hassin très ouvert présente une courbe de résonance très peu sélective. Corrélativement, la résonance s'y établit tres rapidement; aussi de tels' bassins seront-ils soumis relativement souvent $\dot{a}$ des mouvements de seiches résonnants.

Ainsi la connaissance du degré de sélectivité des courbes d'agitation apporte une donnée sur la probabilité de résonance dos darses, en fonction de leur degré d'ouverture. Il est cependant nécessaire de connaître également le spectre des ondes excitatrices et leur probabilité de résonance aux abords immédiats du port étudić. 
BIBLIOGRAPHIE

[1] Breser. - Etude théorique de la réflexion de la houle sur certains obstacles.

La Houlle Blanche, $\mathrm{n}^{\circ}$ A, mars 1952.

[2] BIeser, Le Míhautr.. - Aperçus sur la similitude des modèles réduits destinés à l'étude des seiches portuaires.

La Houille Blanche, $\mathrm{n}^{\circ}$ 3, p. 392-407, juillet 1955.

[3] Biesel, Le Méhauté. - Etude théorique de la réflexion de la houle sur certains obstacles. La Houille Blanche, mars-avril 1955.

[4] Breser. - The similitude of scale models for the study of seiches in harbours.

Vo Congrès du Coastal Engineering, septembre 1955.

[5] Boutan. - Appareils pour la mesure des niveaux rapidement variables sur modèle réduit. La Honille Blanche, $\mathrm{n}^{\circ}$ 2, août-septembre 1953.

[6] Honda, Terlada, Yosmida, Istani, - - An investigation on the secondary ondulations of Oceanic Tides.

Journal of the College of Sciences University, Tokio, $n^{\circ} 24,1908$.

[7] Lamö̈n. - Sur l'hydraulique des fleuves à marées. Revue générale de l'Hydraulique, 1936.

[8] Le Múhauré. - Two-Dimensional seiche in a basin subjected to ineident waves.

$V^{0}$ Congrès du Coastal Engineering, septembre 1954.

[9] Mc Nows. - Sur l'entretien des oscillations des caux portuaires sous l'action de la haute mer. Publications scientifiques et techniques $d u$ Ministere de l'Air, $\mathrm{n}^{\circ} 278,1953$.
[10] Neumanv. - Uber Resonanzschwingungen von Meeresbuchten und die Mündungskorrektur bei Seiches.

Deutsche Hydrographische Zeitschrift, juin 1948.

[11] Provdman. - Dynamical Oceanography. Nethuen and Co Ltd, London, 1945.

[12] RANSFord. - A wave machine of a novel type. International Association for hydratlies Research, Proceedings, Minnesota, septembre 1953.

[13] Vanoni et Carr. - Harbor surging. Proceedings of the first conference on Constal Enginering, 1950.

[14] Varembors. - Etude de l'action d'ouvrages résonnants sur la propagation de la houle. International Association for hydraulics Research, Proceedings, Minnesota, septembre 1953.

[15] Kravtchenko. - SAnton. - Quelques recherches récentes sur les oscillations des eaux portuaires. $6^{c}$ Congrès A.I.R.H, La Haye, 1955.

[16] Apté. - Recherche des mouvements des liquides pesants à surface libre. Thèse, Université de Grenoble.

[17] Le MÉhauté. - Perméabilité des ouvrages maritimes aux ondes de gravité périodiques. IVes Journées de l'Hydraulique, Paris, 1956. Question III. Rapport $\mathrm{n}^{\circ} 4$.

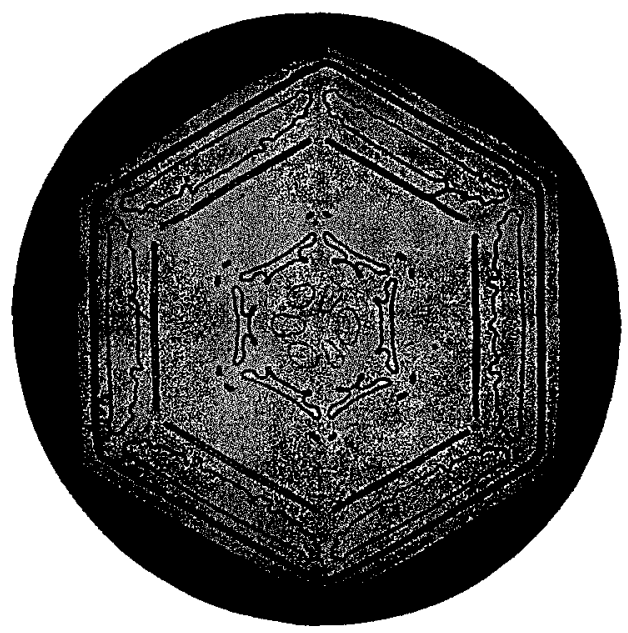

